\title{
Allopurinol alters the expression of cytokines and mediators of immune cells in the systemic 2 tissue by decreasing plasma urate.
}

Lundrim s. Marku

Ismarku@mix.wvu.edu

Follow this and additional works at: https://researchrepository.wvu.edu/etd

Part of the Poultry or Avian Science Commons

\section{Recommended Citation}

Marku, Lundrim s., "Allopurinol alters the expression of cytokines and mediators of immune cells in the systemic 2 tissue by decreasing plasma urate." (2020). Graduate Theses, Dissertations, and Problem Reports. 7724.

https://researchrepository.wvu.edu/etd/7724

This Thesis is protected by copyright and/or related rights. It has been brought to you by the The Research Repository @ WVU with permission from the rights-holder(s). You are free to use this Thesis in any way that is permitted by the copyright and related rights legislation that applies to your use. For other uses you must obtain permission from the rights-holder(s) directly, unless additional rights are indicated by a Creative Commons license in the record and/ or on the work itself. This Thesis has been accepted for inclusion in WVU Graduate Theses, Dissertations, and Problem Reports collection by an authorized administrator of The Research Repository @ WVU. For more information, please contact researchrepository@mail.wvu.edu. 
Allopurinol alters the expression of cytokines and mediators of immune cells in the systemic 2 tissue by decreasing plasma urate.

Lundrim s. Marku

Follow this and additional works at: https://researchrepository.wvu.edu/etd

Part of the Poultry or Avian Science Commons 
Allopurinol alters the expression of cytokines and mediators of immune cells in the systemic tissue by decreasing plasma urate.

Lundrim Marku

Thesis submitted to the Davis College of Agriculture, Natural Resources and Design at West Virginia University

In partial Fulfillment of the requirements for the degree of Master of Science in Animal Physiology

\author{
Hillar Klandorf, Ph.D, Chair \\ Ashok Bidwai, Ph.D, \\ Jianbo Yao, Ph.D, \\ Department of Animal and Nutritional Sciences, West Virginia University,
}

\title{
Morgantown, West Virginia
}

2020

Keywords: Allopurinol, Chickens, Il-6, Tnf-alpha, brain inflammation. Gut inflammation.

Copyright 2020: Lundrim Marku 


\begin{abstract}
Allopurinol alters the expression of cytokines and mediators of immune cells in the systemic tissue by decreasing plasma urate.

\section{Lundrim Marku}

Diminished levels of urate have been linked to oxidative stress in birds and mammals. Urate, a major antioxidant that lowers reactive oxygen/nitrogen species (ROS/RNS), is thought to ameliorate the effects. The relationship between reduced urate, the immune system and the pathogenesis of the intestine, the liver or the brain has not been well characterized in avians. Moreover, recent studies have proposed that an increased permeability in the intestine due to some insult can induce inflammation in peripheral organs such as the brain. Allopurinol, a relatively toxic purine analogue that serves as a xanthine oxidase inhibitor, reduces urate levels which can subsequently induce an inflammation state in the intestine. For this study, White Leghorn Chickens $(n=44)$ were divided into 11 groups, which constituted of six control groups and five allopurinol treatments fed at $15 \mathrm{mg} / \mathrm{kg}$ body weight. The dose of allopurinol was increased $5 \mathrm{mg} / \mathrm{kg}$ body weight each week for 12 weeks. Blood samples were obtained by from the brachial vein of the wing. Liver panel blood chemistries were performed to characterize the inflammations state. At the end of the study, tissues were removed after cervical dislocation and placed in liquid nitrogen. Expression levels of TNF- $\alpha$, IL-6, FASLG and COX-2 were subsequently investigated in the intestines, liver and midbrain. The results showed that urate was reduced in all treatment groups regardless of time $(\mathrm{p}<0.05)$, treatment groups exhibited reduced amounts of bile acids throughout time $(\mathrm{p}<0.05)$ and female treatment groups exhibited increased amounts of LDH and AST from week 2 to the end of the experiment $(\mathrm{p}<0.05)$. The effect of allopurinol on the intestines of females showed that expression of IL-6, COX-2, TNF- $\alpha$ expression increased from every other group by $185.93,211.74$ and 107.52 times respectively ( $\mathrm{p}$ $<0.05)$. TNF- $\alpha$ expression in the liver decreased in both females and males by 3 and 2 -fold respectively ( $\mathrm{p}<0.05)$. FASLG expression in the liver decreased 2 -fold in both male and females respectively ( $\mathrm{p}<0.05$ ). The effect of allopurinol on the midbrain of females showed that IL-6 expression decreased by 0.061 fold $(?)(\mathrm{p}<0.05)$ whereas in males IL-6 expression increased by almost 2 -fold $(\mathrm{p}<0.05)$. COX-2 expression in the midbrain increased in females and males by 7 and 10 times ( $p<0.05)$. The midbrain of treated females reveal that the inflammatory potential was reduced in the brain tissue of female chickens due to decreased IL-6 expression. Females were found respond more intensely to allopurinol administration from an increase of intestinal permeability which could alter the laying quality and reproductive fitness. Lastly, an upregulation of IL-6 in males also induce inflammation and could be used to create a model of neuropathological disorders. 


\section{Table of Contents}

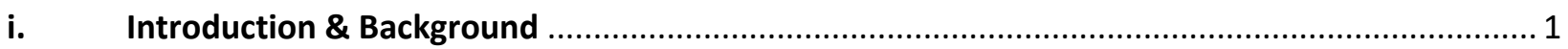

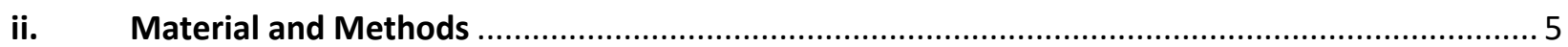

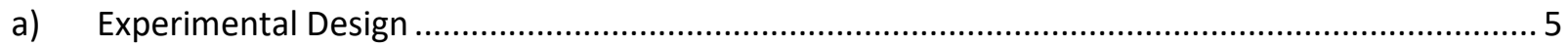

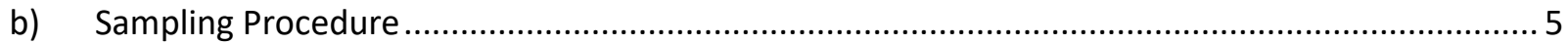

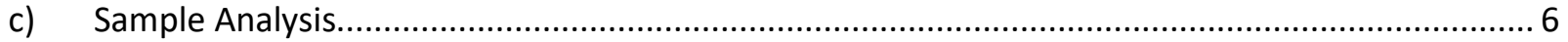

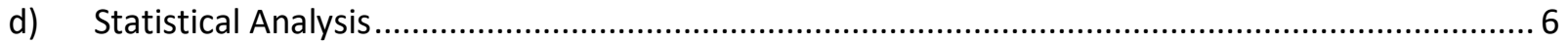

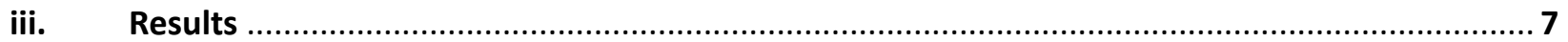

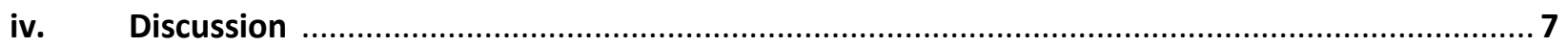

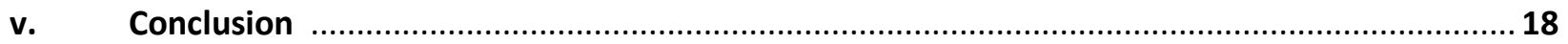

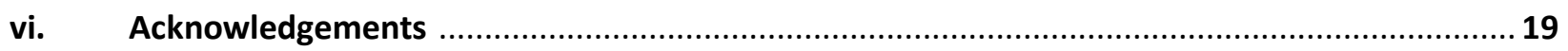

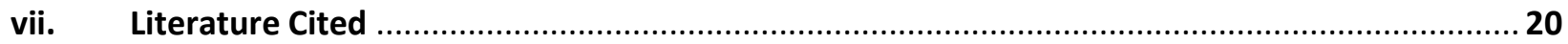




\section{i. Introduction}

Urate is synthesized the liver, intestines and the vascular endothelium as a product of an exogenous pool of purines [1]. Urate can also be produced endogenously by damaged, dying or dead cells; where nucleic acids, adenine, and guanine, are degraded into urate to act as damageassociated molecular patterns [1], [2]. This biomolecule conventionally generates concerns due to acute and chronic inflammatory arthritis, gout, and other metabolic diseases [1], [3]. However, it is also thought to have a dual role and serve by inducing a type 2 immune response [1], [2], [4]. The inhibition of urate production can elucidate the protective potential by observing how lowered urate concentrations alter immunological function in several organs [5], [6]. Several studies have indicated how the intestinal environment can exert profound effects on the liver and central nervous system through the regulation of the microbiota and the intestinal barrier function [7]. This gut-brain connection is becoming a model of immune activity with a fundamental contribution towards neurodegenerative disorders [7]. These studies indicate that inflammation in the intestine appears to be particularly relevant in the disease pathogenesis.

Studies have shown that urate serves as a potent scavenger of singlet oxygen, peroxyl radicals and hydroxyl radicals [6]. Elevated urate concentrations in the circulation helps to protect cells by scavenging these free radicals which then prolongs the organism's life [6], [8]. Living systems have adapted to regulate free radicals by developing pathways to inactivate these reactive species such as oxygen and nitrogen, which induce tissue injury [6], [9]. Allopurinol, a toxic purine analog, serves as a xanthine oxidase inhibitor which reduces urate concentrations; which can induce a type I inflammation state in the intestine and the brain of birds [3]. Changes

in the compositions of the bacterial populations in the intestines have also been widely associated with an array of conditions that can cause neurological and developmental disorders such as 
multiple sclerosis, autism, depression, schizophrenia, and Parkinson's disease [4], [7].

Shifts in intestinal microbiota can alter levels of growth factors and signaling proteins in the brain, which contributes to functional changes in the remodeling and inflammation?[5]. Among the roles for gut bacteria are the conversion of primary bile acids produced by the liver to secondary bile acids which then are absorbed through the intestinal epithelium [7]. Moreover, bile acids can also act as potent signaling molecules that regulate a variety of processes related to both the nervous and immune systems. A detailed look into the effects of allopurinol on the early recognition and effector response by the immune system in the intestines could elucidate how these responses affect the liver and brain [4], [7]. Metabolites generated from intestinal microbes such as those described here have also been reported to alter host gene expression in the brain, providing ways for the microbiota to influence the activity of the CNS [4], [7]. Thus, the administration of allopurinol can evoke strong type 1 immune reactions via IL- 6 by altering the intestinal environment and induce effects that ultimately alter CNS function.

\section{The Immune System and The Urate Paradox}

A vast amount literature shows an elevated level of urate is strongly associated with inflammatory diseases such as hypertension, cardiovascular and cerebrovascular events [2], [3], [10]. While urate does account for over half of the free radical scavenging activity in blood [8], it can also be thought to combat the oxidative stress implicated in several neurodegenerative diseases. Antioxidant activities of urate can quench superoxide and singlet oxygen and protecting oxidation of vitamin $\mathrm{C}$ through the chelation of iron [6], [11]. These qualities make urate an attractive CNS antioxidant because neurons are remarkably susceptible to oxidative stress. In multiple sclerosis, free radicals can contribute to the inflammation and demyelination of axons [10], [12]. Thus, preventing oxidative damage may delay onset and improve the prognosis of 
CNS disorders [10]. The ratio of reactive species over antioxidants determine the shift from their advantageous function to detrimental effects [11]. The major source of these reactive species that become detrimental are dependent on cell type, duration of oxidant production, reactive species produced, and the localization of their source [6]. The oxidant-antioxidant paradox can be further investigated by the analyzing how decreased blood urate can alter various genes associated with inflammation [4], [6], [10].

\section{Urate's Systemic Effects}

Immune cells can engage in direct communication with these dying cells as well as with neurons. The extent of the functional impact of neuroimmune synapses is not known. However, activated immune cells can modulate neuronal activity by using neurotransmitters and cytokines [4]. Proinflammatory cytokines and activated immune cells in the circulation also access the brain when the blood brain barrier is compromised [2]. Systemic inflammation associated with increased BBB permeability can be considered a precursor to neurodegenerative diseases [13]. Extensive evidence has reported linking molecules associated with inflammatory conditions, which include cytokines, reactive oxygen species, matrix metalloproteases, and mediators of angiogenesis with blood brain barrier disruption [2], [13]. Additionally, a positive feedback loop involving IL-6 in conjunction with neuroimmune reflex circuits has been implicated in increasing permeability such that peripheral T cells gain access to the CNS [7]. Leaks in the blood brain barrier can significantly alter immune responses to CNS antigens and compromise CNS protection against potentially harmful substances [7], [13].

The systemic effects of intestinal inflammation may be further augmented by increases in intestinal permeability via some insult to the gut [7]. Acute tissue injury may occur with a severe infection generated by an intestinal pathogen, which causes temporary defects in the intestinal 
epithelial barrier [2]. These low-grade insults induce more selective increases in paracellular permeability through regulation of tight junctions. Intestinal microbes also regulate expression of barrier promoting tight junction proteins [7]. While many proinflammatory cytokines secreted by activated immune cells, which include TNF, IL-1 $1 \beta$, and IL-6, allow for tight junctions to increase barrier permeability in order to facilitate recruitment of immune cells and other molecules [2], [14], [15], [16], [17], [18]. However, a side effect of this response permits microbes to leak from the intestine into the peritoneal cavity and from there into the blood, which then triggers a systemic proinflammatory immune responses [7].

Typically, the immune challenge is rapidly cleared, proinflammatory responses terminate, and gut barrier function is restored [7]. However, unique features of the intestine allow for persistent inflammation and barrier dysfunction [7]. Sustained permeability of the intestinal barrier can have harmful effects on numerous body systems. Many microbial components can trigger "leaky gut syndrome" and lead to conditions like Irritable bowel syndrome (IBS) and metabolic syndrome. Moreover, recent studies have implicated that intestinal permeability can be with linked with CNS dysfunction, which include Parkinson's, Alzheimer's, autism, schizophrenia, multiple sclerosis, depression, anxiety, and post-traumatic stress disorder [7], [7]. Proinflammatory responses in the brain can alter CNS function and CNS immune responses can have serious and enduring consequences. If inflammation becomes chronic, proinflammatory cytokines and oxidative stress that are linked to neuron death, and neuroinflammation should be investigated as a key factor in numerous neurodegenerative diseases. 


\section{ii. Materials and Methods}

All procedures and experiments were approved by West Virginia University's Institution Animal Care and Use Committee 1708009034.

\section{Experimental Design}

Chickens were housed within pens and maintained under a twelve-hour light-dark cycle with temperature set at $27^{\circ} \mathrm{C}$. One dose of allopurinol will be administered $(15 \mathrm{mg} / \mathrm{kg}$ of body weight) as a feed additive to chickens. The chickens were allowed a growth period of two to three weeks before the start of treatments. On the third week of age, the chickens will be assigned at random into treatment groups, Control diet and Allopurinol in the diet. The treatment period will be 12 weeks until euthanasia. Three weeks after growth, chickens $(n=44)$ were divided into 11 groups and tagged with a leg band. The chickens were maintained on a starter diet before the experiment began. The leghorn chickens were started on their respective treatment's allopurinol were purchased from Sigma-Aldrich (Saint Louis, MO, USA). Food and water were provided ad libitum. At 12 weeks of age the birds were killed by cervical dislocation and the intestines, liver and midbrain tissues were removed and stored in liquid nitrogen prior to analysis.

\section{Sampling procedure}

Blood samples were obtained from the brachial wing vein of 2 birds per pen at week two of treatment for twelve weeks. Each blood sample was placed in a heparinized tube and stored on ice. The blood samples were centrifuged at $2000 \mathrm{rpm}$ for 20 minutes at $4^{\circ} \mathrm{C}$. Plasma samples were then transferred to micro-centrifuge tube and stored in a $-80^{\circ} \mathrm{C}$ freezer pending analysis. 


\section{Sample analysis}

Plasma uric acid (PUA) was measured using Infinity ${ }^{\mathrm{TM}}$ Uric Acid Liquid Stable Reagent assay from Thermo Scientific (Waltham, MA, USA). Liver Blood panel chemistries were performed with the help of Dr. Jesse Fallon at the Cheat Lake Veterinary Hospital.

\section{RNA Extraction, cDNA Synthesis and qPCR Total RNA}

The primers used in these experiments can be referred to Table 1. Total RNA was isolated from tissues using the TRIZOL Reagent (Invitrogen, Carlsbad, CA). To quantify expression levels, equal amounts of cDNA were synthesized using the Advantage RT-for-PCR kit (Clontech, Mountain View, CA). GAPDH was amplified as an internal control. qPCR was performed using Power SYBR Green PCR Master Mix (Applied Biosystems). All qPCR was performed using SYBR Green, conducted at $50^{\circ} \mathrm{C}$ for 2 minutes, $95^{\circ} \mathrm{C}$ for 10 minutes, and then 40 cycles of $95^{\circ} \mathrm{C}$ for 15 seconds and $60^{\circ} \mathrm{C}$ for 1 minutes. The specificity of the reaction was verified by melt curve analysis. qPCR was conducted at $95^{\circ} \mathrm{C}$ for 10 minutes, followed by 40 cycles of $95^{\circ} \mathrm{C}$ for 15 seconds and $60^{\circ} \mathrm{C}$ for 1 minutes. The threshold crossing value was noted for each transcript and normalized to the internal control. The relative quantitation of each mRNA was performed using the comparative delta delta $\mathrm{Ct}$ method. Experiments were performed using an ABI Prism 7900 System (Applied Biosystems), and data processing was performed using ABI SDS v2.1 software (Applied Biosystems).

\section{Statistical Analysis}

The statistical program SAS (version 14.3; SAS Institute Inc., Cary, NC) was used to analyze the data. Both time and treatment effects were analyzed. Weeks were considered as a fixed effect whereas birds were treated as random effect. A generalized linear model was used to study the treatment effect at week 12 of the study. Values are expressed as least square (LSM) \pm 
standard error (SE) unless stated otherwise. JMP and SAS software (JMP®, Version Pro 14.0, SAS Institute Inc., Cary, NC, Copyright @2015; SAS®, Version 9.4, SAS Institute Inc., Cary, NC, Copyright (C2002-2012). Significance criterion alpha for all tests was set to 0.05.

\section{iii. Results}

\section{Plasma Urate and Liver Panel}

The results showed that plasma urate was reduced in all treatment groups regardless of time $(\mathrm{p}<0.05)($ Figure $1 \mathrm{~K})$. Treatment groups exhibited reduced amounts of bile acids throughout the study period $(\mathrm{p}<0.05)($ Figure $1 \mathrm{~L})$ while female treated birds exhibited increased amounts of LDH and AST over time ( $<$ 0.05) (Figure 1D and 1J).

\section{Intestines}

In female chicks, allopurinol increased IL-6, COX-2, TNF- $\alpha$ expression by 186, 212 and 108 times respectively $(\mathrm{p}<0.05)($ Figure $2 \mathrm{~A}-\mathrm{C})$ with the combination of sex and treatment found to be significant $(\mathrm{p}<0.05)$ (Figure 2A). Tukey HSD, teste done to determine if there is an honest significant difference of LSM differences revealed that IL-6 expression was increased in females that were fed the allopurinol treatment $(p<0.05)$.

\section{$\underline{\text { Liver }}$}

The effect of allopurinol on TNF- $\alpha$ expression was decreased in both females and males by 3 and 2-fold compared to their respective control groups ( $p<0.05)$ (Figure 3a). The effect of allopurinol on FASLG expression in the liver was decreased by 2 times in both male and females from their respective control groups $(\mathrm{p}<0.05)$ (Figure 3b).

\section{$\underline{\text { Midbrain }}$}

Allopurinol administration to female chickens decreased IL-6 expression from its 
respective control to a fold change of $0.061(\mathrm{p}<0.05)$ (Figure 4a) with the combination of sex and treatment also found to be significant $(\mathrm{p}<0.05)$. Allopurinol administration to male chickens increased IL-6 expression from the respective controls by 1.98 times $(\mathrm{p}<0.05)$ (Figure 4a) with the combination of sex and treatment was found to be significant $(\mathrm{p}<0.05)$. Tukey HSD of LSM differences revealed that females with the allopurinol treatment have statistically decreased from every other group. Males were also found to statistically decrease from every other group with the control groups of both sexes statistically similar to each other. Allopurinol increased COX-2 expression in the midbrain in both females and males by 7 and 10 -fold compared to their respective control groups $(\mathrm{p}<0.05)$ (Figure $4 \mathrm{~b})$.

Figure 1A. Albumin-to-Globulin Ratio. Total plasma protein concentrations in female treated chickens $(n=11)$ female treated chickens $(n=11)$ at 2, 6, 12 wks of age; Male treated chickens $(n=11)$ at 2, 6, 12 wks of age; Control Females $(n=11)$ at 2, 6, 12 wks of age. Control Males $(n=11)$ at 2, 6, 12 wks of age.

Figure 1B. Albumin Concentrations. Total albumin concentrations in g/dL. Female treated chickens $(n=11)$ female treated chickens $(n=11)$ at 2, 6, 12 wks of age; Male treated chickens $(n=11)$ at 2, 6, 12 wks of age; Control Females $(n=11)$ at 2, 6, 12 wks of age. Control Males $(n=11)$ at 2, 6, 12 wks of age.

Figure 1C. Alkaline Phosphatase. Alkaline phosphatase concentrations in IU/L. Female treated chickens $(n=11)$ female treated chickens $(n=11)$ at 2, 6, 12 wks of age; Male treated chickens $(n=11)$ at 2, 6, 12 wks of age; Control Females $(n=11)$ at 2, 6, 12 wks of age. Control Males $(n=11)$ at 2, 6, 12 wks of age.

Figure 1D. Aspartate Aminotransferase. Aspartate aminotransferase concentrations in 
IU/L. Female treated chickens $(\mathrm{n}=11)$ female treated chickens $(\mathrm{n}=11)$ at 2, 6, 12 wks of age; Male treated chickens $(n=11)$ at 2, 6, 12 wks of age; Control Females $(n=11)$ at 2, 6, 12 wks of age. Control Males ( $\mathrm{n}=11)$ at 2, 6, 12 wks of age. Female treated birds exhibited increased amounts of AST over time $(\mathrm{p}<0.05)$.

Figure 1E. Creatine Kinase. Creatine kinase concentrations in IU/L. Female treated chickens $(\mathrm{n}=11)$ female treated chickens $(\mathrm{n}=11)$ at 2, 6, 12 wks of age; Male treated chickens $(\mathrm{n}=11)$ at 2, 6, 12 wks of age; Control Females $(\mathrm{n}=11)$ at 2, 6, 12 wks of age. Control Males $(\mathrm{n}=11)$ at 2, 6, 12 wks of age.

Figure 1F. Globulin. Globulin concentrations in g/dL. Female treated chickens $(\mathrm{n}=11)$ female treated chickens(n=11) at 2, 6, 12 wks of age; Male treated chickens $(n=11)$ at 2, 6, 12 wks of age; Control Females $(\mathrm{n}=11)$ at 2, 6, 12 wks of age. Control Males $(\mathrm{n}=11)$ at 2, 6, 12 wks of age.

Figure 1G. Phosphorus. Phosphorus concentrations in mg/dL. Female treated chickens $(\mathrm{n}=11)$ female treated chickens $(\mathrm{n}=11)$ at 2, 6, 12 wks of age; Male treated chickens $(\mathrm{n}=11)$ at 2, 6, 12 wks of age; Control Females $(\mathrm{n}=11)$ at 2, 6, 12 wks of age. Control Males $(\mathrm{n}=11)$ at $2,6,12 \mathrm{wks}$ of age.

Figure 1H. Blood Urea Nitrogen. BUN. Female treated chickens $(n=11)$ female treated chickens(n=11) at 2, 6, 12 wks of age; Male treated chickens $(\mathrm{n}=11)$ at 2, 6, 12 wks of age; Control Females $(n=11)$ at 2, 6, 12 wks of age. Control Males $(n=11)$ at 2, 6, 12 wks of age.

Figure 1I. Total Protein. Total protein concentrations in g/dL. Female treated chickens $(\mathrm{n}=11)$ female treated chickens $(\mathrm{n}=11)$ at 2, 6, 12 wks of age; Male treated chickens $(\mathrm{n}=11)$ at 2, 6, 12 wks of age; Control Females $(n=11)$ at 2, 6, 12 wks of age. Control Males $(n=11)$ at 2, 
6,12 wks of age.

Figure 1J. Lactate Dehydrogenase. LDH concentrations in IU/L. Female treated chickens $(\mathrm{n}=11)$ female treated chickens $(\mathrm{n}=11)$ at 2, 6, 12 wks of age; Male treated chickens $(\mathrm{n}=11)$ at 2, 6, 12 wks of age; Control Females $(\mathrm{n}=11)$ at 2, 6, 12 wks of age. Control Males $(\mathrm{n}=11)$ at 2, 6, 12 wks of age. Female treated birds exhibited increased amounts of LDH over time $(\mathrm{p}<0.05)$.

Figure 1K. Urate. Urate concentrations in $\mathrm{mg} / \mathrm{dL}$. Female treated chickens $(\mathrm{n}=11)$ female treated chickens(n=11) at 2, 6, 12 wks of age; Male treated chickens $(n=11)$ at 2, 6, 12 wks of age; Control Females $(\mathrm{n}=11)$ at 2, 6, 12 wks of age. Control Males $(\mathrm{n}=11)$ at 2, 6, 12 wks of age. Plasma urate was reduced in all treatment groups regardless of time $(\mathrm{p}<0.05)$.

Figure 1L. Bile Acids. Bile Acids concentrations in $\mu$ mol/l. Female treated chickens $(\mathrm{n}=11)$ female treated chickens $(\mathrm{n}=11)$ at 2, 6,12 wks of age; Male treated chickens $(\mathrm{n}=11)$ at 2, 6, 12 wks of age; Control Females $(n=11)$ at 2, 6, 12 wks of age. Control Males $(n=11)$ at 2, 6,12 wks of age. Treatment groups exhibited reduced amounts of bile acids throughout time $(\mathrm{p}<$ $0.05)$.

\section{qPCR}

Table 1. Oligonucleotide primers for qPCR

\begin{tabular}{lllll}
\hline Gene & Forward $\left(5^{\prime}-3^{\prime}\right)$ & Reverse $\left(5^{\prime}-3^{\prime}\right)$ & Annealing $\left({ }^{\circ} \mathrm{C}\right)$ & Accession \\
\hline *GAPDH & Ggtggccatcaatgatccet & Ccgttctcagccttgacagt & 60 & NC_006088.5 \\
TNF-alpha & Gagatcctcctgcactgaa & Cacatcccetttctggaaga & 60 & NC_006101.5 \\
FASLG & Tgctggacctcgtttagctt & Ctcatttccactgccatct & 60 & NC_006093.5 \\
IL-6 & Aatggtgggggtcatatcaa & Agtcacgtttgatggcttcc & 60 & NC_006089.5 \\
OCLN & Cgagttggatgagtcccagta & Ggtgtcgaactcctgcttgta & 60 & NC_006115.5
\end{tabular}




\begin{tabular}{lllll} 
COX-2 & Tcetcatgttcctgagcatct & Gctcctgttcaagagctcac & 60 & NC_006095.5 \\
KRT18 & Gaactactgggacaccatcca & Atgcctcatacttcaccetga & 60 & NC_008465.4 \\
CRP & Tcttctcctacgccaccaaa & Acgcggaaggtgacgtattt & 60 & NC_006112.4 \\
\hline
\end{tabular}

Figure 2a. The effect of allopurinol on IL-6 expression in the intestines of allopurinol fed chickens and healthy controls. IL6 mRNA was normalized to that of GAPDH. Female treated chickens ( $(n=3)$; Male treated chickens $(n=3)$; Control Females $(n=3)$. Control Males $(n=3)$. The treated female group had an LSM=185.93 and SE=32.74. The F-ratio was 7.25 and the pvalue $=0.0376$ using an ANOVA test. The combination of sex and treatment was found to be significant with females at t-Ratio 2.72 and $p$-value $=0.0263$. Tukey HSD of LSM differences show that females with the allopurinol treatment IL-6 expression are statistically different from every group.

Figure 2b. The effect of allopurinol on COX-2 expression in the intestines of allopurinol fed chickens and healthy controls. COX-2 mRNA was normalized to that of GAPDH. Female treated chickens $(n=3)$; Male treated chickens $(n=3)$; Control Females $(n=3)$. Control Males $(\mathrm{n}=3)$. The treated female group had an LSM=211.74 and SE=18.144. The F-ratio was 11.909 and the $\mathrm{p}$-value $=0.0376$ using an ANOVA test. The combination of sex and treatment was found to be significant with females at t-Ratio 4.31 and $\mathrm{p}$-value $=0.0191$.

Figure 2c. The effect of allopurinol on TNF- $\alpha$ expression in the intestines of allopurinol fed chickens and healthy controls. TNF- $\alpha$ mRNA was normalized to that of GAPDH. Female treated chickens $(n=3)$; Male treated chickens $(n=3)$; Control Females $(n=3)$. Control Males $(\mathrm{n}=3)$. The treated female group had an LSM=211.74 and SE=18.144. The F-ratio was 4.58 and the $p$-value $=0.0376$ using an ANOVA test. The treatment was found to be significant at a -Ratio 
2.68 and $\mathrm{p}$-value $=0.0281$.

Figure 3a. The effect of allopurinol on TNF- $\alpha$ expression in the liver of allopurinol fed chickens and healthy controls. TNF- $\alpha$ mRNA was normalized to that of GAPDH. Female treated chickens $(n=3)$; Male treated chickens $(n=3)$; Control Females $(n=3)$. Control Males $(n=3)$. TNF- $\alpha$ expression of treated groups was statistically different from the control groups. The F-ratio was 4.33 and the p-value=0.0432 using an ANOVA test. All groups were found to similar when analyzing with Tukey-HSD.

Figure 3b. The effect of allopurinol on FASLG expression in the liver of allopurinol fed chickens and healthy controls. FASLG mRNA was normalized to that of GAPDH. Female treated chickens $(n=3)$; Male treated chickens $(n=3)$; Control Females $(n=3)$. Control Males $(n=3)$. FASLG expression of treated groups was statistically different from the control groups. The treatment was found to be significant at a t-Ratio 5.10 and p-value=0.0476. All groups were found to similar when analyzing with Tukey-HSD.

Figure 4a. The effect of allopurinol on IL-6 expression in the midbrain of allopurinol fed chickens and healthy controls. IL6 mRNA was normalized to that of GAPDH. Female treated chickens $(n=3)$; Male treated chickens $(n=3)$; Control Females $(n=3)$. Control Males $(n=3)$. The effect of allopurinol on female chickens on IL-6 expression is statistically different from every other group with an LSM=0.061 and $\mathrm{SE}=0.031$. The effect of allopurinol on male chickens on IL-6 expression is statistically different from every other group with an LSM=1.98 and $\mathrm{SE}=0.0312$. The control groups of IL-6 expression is statistically different from every other group with females and males respectively $\mathrm{LSM}=1.089$ and $\mathrm{LSM}=1.219$ and $\mathrm{SE}=0.031$. The Fratio was 6.392 and $p$-value $=0.016$ using an ANOVA test. The combination of sex and treatment was found to be significant with females at t-Ratio -2.87 and $p$-value $=0.021$. Tukey HSD of 
LSM differences show that females with the allopurinol treatment IL-6 expression are statistically different from every other group. Males were also found to be statistically different from every other group; while the control groups were statistically different every other group.

\section{Figure 4b. The effect of allopurinol on COX-2 expression in the midbrain of allopurinol fed} chickens and healthy controls. COX-2 mRNA was normalized to that of GAPDH. Female treated chickens $(n=3)$; Male treated chickens $(n=3)$; Control Females $(n=3)$. Control Males $(n=3)$. The effect of allopurinol on COX-2 expression is statistically different from the control regardless of sex. The treatment was found to be significant at a t-Ratio 2.34 and pvalue $=0.0476$. All groups were found to similar when analyzing with Tukey-HSD.

\section{iv. Discussion:}

Urate was found to be reduced in all treatment groups regardless of time $(\mathrm{p}<0.05)$ indicating that the allopurinol treatment generated an inflammatory state [12]. Liver panels were analyzed to determine if allopurinol could induce toxicity by analyzing major biomarkers. Treatment groups of both male and females revealed reduced amounts of bile acids throughout the study ( $\mathrm{p}$ 0.05), which suggest an inflammation state in both the intestinal tract and liver [7]. Lower concentrations of bile acids reduce cholesterol solubility, which leads to microcrystal formation and subsequently the formation of gallstones [7]. Female treated groups exhibited increased amounts of LDH and AST over time ( $\mathrm{p}<0.05)$. The high levels of LDH in the females suggests some form of tissue damage, which could develop into severe disease over time. In addition, the high AST levels also serves as a sign of liver damage and immune system dysfunction [19], [20]. It is also important to note that albumin and globulin ratios were not altered which indicates that kidney and liver condition is still viable and able to function. Alkaline phosphatase function was not compromised which indicates that the breakdown of 
proteins was not altered, and subsequent liver diseases or bone disorder were not prevalent. Heart condition was not found to induce failure as indicated by creatine kinases. Total protein was not found to be different which indicates rates of clearance and production were not altered. BUN was found to be unaltered which indicated the kidneys are functioning well. IN addition, phosphorus also monitors kidney function but also bone and liver disease. No changes were found in the preceding.

\section{$\underline{\text { Intestines }}$}

The effect of allopurinol administration on the duodenal section of the small intestine was evaluated by examining the expression levels of IL-6, IL-8, FASLG, CCL5, OCLN, TJP1 and TNF- $\alpha$. Allopurinol treatment increased the expression of IL-6, COX-2, TNF- $\alpha$ in females by 186, 212 and 108-fold respectively. Intestinal epithelial cells (IECs) form a highly organized cellular system. Analysis of the intestines is important because the system is maintained in a dynamic steady state by proliferating and differentiating cells [2], [21]. The cells that originate from the crypts of Lieberkühn have multiple tasks that participate in digestion, absorption, and protection [21]. All IECs have been shown to actively defend the epithelial surface, and aid in the recruitment of immune cells by expressing and secreting pro-inflammatory cytokines and chemokines [7], [21]. The intestinal cross talk uses growth factors, cytokines, chemokines, and extracellular proteins between IECs and other cell types to maintain homeostasis [2], [7].

Interleukin 6 exerts multiple functions in the body and serves as indicator of intestinal inflammation with pleiotropic functions including both a pro-inflammatory and a trophic factor [4], [14], [15], [22]. Within the intestine, IL-6 has been shown to prevent epithelial apoptosis during prolonged inflammation while inducing intraepithelial lymphocytes to undergo epithelial proliferation and wound repair at the site of an inflammatory injury [14], [15], [22]. The 186-fold 
increase of IL-6 in the gut is suggestive of significant immune recruitment and trophic functions of IECs resulting from the initial insult generated from allopurinol administration. Additionally, a secondary response could occur to combat infection from microbiota in treated females. Females may have had a greater induced response due to synergism with to egg laying [23]. The previous study investigated how probiotics and agents that induce gut sustainability and the strengthen of the gut barrier would increase the capability, quality of the eggs and the overall health of females. Which could also warrant reproductive studies to determine the toxicology of allopurinol and how laying cycles alter reproductive hormones concentrations. An overactive immune system could be working to correct the initial insult and protect the offspring by over expressing IL-6 for wound recovery, cell proliferation and the introduction of lymphocytes [9], [7], [15], [22].

COX-2 converts arachidonate to prostaglandin $\mathrm{H} 2$ and is constitutively expressed in the endothelium, kidney, brain and cancers [23]. COX-2 is responsible for the production of inflammatory prostaglandins and the upregulation associated cell adhesion, resistance to apoptosis, and tumor angiogenesis [7], [21], [22]. The 212-fold increase of COX-2 in the intestines is thus suggestive of the upregulation of cell adhesion to prevent the blood barrier from deteriorating, especially in females in order to ensure reproductive success associated with egg lay. Lastly, TNF- $\alpha$ is regulator of both the immune response and inflammation. TNF- $\alpha$ is expressed by macrophages, T-cells and NK cells, which causes both inflammation and endothelial activation [7], [15], [21], [22]. The 108-fold increase from the intestines suggests recruitment of the lymphocytes listed above, resulting in inflammation and endothelial activation in order to remodel the deteriorating regions from the toxic effects of allopurinol [4]. Allopurinol as a chemical agent is shown to induce gut instability and the weaken of the gut barrier, alter the 
quality of the eggs and the overall fitness of females [23]. An overactive immune system is working to ameliorate these effects by inducing wound recovery. Which could also warrant reproductive studies to determine the toxicology of allopurinol on how of reproductive hormones are being alter in laying cycles concentrations [23].

\section{$\underline{\text { Liver }}$}

The effect of allopurinol administration on the liver was evaluated by examining expression levels of IL-6, FASLG, CCL5, CRP, KRT-18, OCLN, TJP1 and TNF- $\alpha$. The liver is the target organ for many toxic chemicals and plays a central role in detoxification and elimination [17], [19], [24]. However, when chemical or biological compounds overload the livers' functional capacity to clear the drug or infection, it begins to fail [14], [20], [24]. Allopurinol treatment decreased TNF- $\alpha$ expression in the liver in both females and males by 3 and 2-fold compared to the respective control groups while FASLG expression decreased 2-fold in both male and females compared to their respective controls. The Fas receptor/ligand system is the most important apoptotic initiator in the liver [20]. Dysregulation of this pathway can contribute to abnormal cell proliferation, cell death and prevent the immune system from targeting cancer cells [20], [24]. The findings suggest that the treated groups did not have any decrease in hepatocyte homeostatic and mitogenic activity, which could have led to liver damage and other associated pathologies. However, the findings are counterintuitive to the results from the liver panel which suggested that the low levels of bile acids, increased LDH and AST indicated liver damage. Moreover, the expression of KRT18, a biomarker for cell damage analogous to $\mathrm{CK}$ in heart failure, was also not shown to have any significant changes [24].

\section{$\underline{\text { Midbrain }}$}

The effect of allopurinol administration on the midbrain was evaluated by examining 
expression levels of IL-6, FASLG, CCL5, OCLN, TJP1 and TNF- $\alpha$. Interleukin-6 plays a critical role in the pathogenesis of inflammatory disorders and in the physiological homeostasis of neural tissue [7], [13], [25]. Profound neuropathological changes such as multiple sclerosis, Parkinson's and Alzheimer's disease are associated with increased IL-6 expression in brain [7], [13], [25]. Allopurinol administration to the egg laying female chickens decreased IL-6 expression compared to its respective controls to a fold change of 0.061 with the combination of sex and treatment found to be significant $(\mathrm{P}<0.05)$. These data suggest that allopurinol reduced both the remodeling potential and inflammation state in these birds [26]. However, allopurinol increased IL-6 expression in adult male chickens compared to their respective controls by 1.98 times with the combination of sex and treatment found to be significant $(\mathrm{P}<0.05)$, which suggests that males were more suspectable to developing inflammation when comparing both treatment groups [26]. The upregulation in IL-6 expression suggests the use of male treated chickens to investigate other neuropathological disorders [7]. However, additional experiments need to be conducted to determine if other inflammatory pathways are also being upregulated could mimic conditions such as multiple sclerosis, Parkinson's and Alzheimer's disease.

COX-2 is an inducible and rapidly expressed gene that responds to growth factors, cytokines, and pro-inflammatory molecules [27], [28]. Since its discovery, COX-2 has been recognized as a major contributor to inflammatory reactions in peripheral tissues that can progress to several acute and chronic diseases [7], [27], [28]. However, in the central nervous system, COX-2 is expressed under normal conditions and contributes to fundamental brain functions, such as synaptic activity, memory consolidation, and functional hyperemia [27], [29]. The gene is thus tightly regulated, as it is triggered and sustained by the activation of microglia [27], [29]. Allopurinol increased COX-2 expression in the midbrain both females and males by 7 
and 10-fold respectively, which suggests that that both treated groups are involved in extensive neuroinflammation and remodeling due low urate levels [29].

\section{v. Conclusion:}

The reduced levels of urate in avians that have been traditionally linked to oxidative stress, now purport investigations on the mechanisms by which urate interacts with systemic organs. Results from the present study suggest that the inflammatory pathways in the intestines of female's chickens respond more intensely to allopurinol administration than males.

Interestingly, allopurinol treatment results in an increase in intestinal permeability due to insult from the drug, which increases the expression of IL-6. Moreover, expression levels of IL-6 in the brain of males suggest that inflammation was induced in the midbrain. The function of neurological remodeling and inflammation associated with it to be increased in both sexes with the upregulation of COX-2 occurring in both sexes. Allopurinol was suggested to induce cellular remodeling of the intestines and generate an inflammation state in due to the upregulation of IL$6, \mathrm{COX}-2$ and TNF- $\alpha$. Which could also warrant reproductive studies to determine the toxicology of allopurinol on how of reproductive hormones are being alter in laying cycles concentrations. Results from the liver panel also suggested an inflammatory state as reduced amounts of bile acids and urate and increased amounts of LDH and AST were measured in females. However, TNF- $\alpha$ and FASLG expression in the liver were decreased in both females and males, which indicates that the inflammation may not have been severe. The midbrain of allopurinol treated females revealed that IL-6 expression decreased, which suggests that allopurinol reduces the inflammatory potential in the brain tissue of female chickens. However, an upregulation in males could indicate that allopurinol induces an increase in IL-6 which could be used to create a model of neuropathological disorders. The increased expression COX-2 also 
bolsters the idea that neuromodulation is upregulated due to treatment. Additional experiments must be conducted in males to determine if other inflammatory pathways are being upregulated that could possibly mimic the conditions associated with the development of multiple sclerosis, Parkinson's and Alzheimer's disease.

Future experiments should involve a redesign of the experiment model by four separate groups: control males, control females, treated males and treated females each with their own feed to strengthen the power of these findings. Another facet that could be improved upon is preforming weekly or biweekly bleeds of all chickens as well as collection of tissues. With these bleeds, additional biomarkers could be measured with liver panels and evaluating genes that are involved with liver toxicity, inflammation and regulatory pathways. Additional proinflammatory proteins that should be investigated include IL-1B, IL-11 and IL-8, whereas regulatory pathways can be further characterized by investigating IL-4, IL-6, IL-10, IL-11, and IL-13. In addition to evaluating mRNA expression levels of these biomarkers, protein levels could be investigated with ELISAs. Lastly, additional biomarkers associated with neurological disorders should be measured to determine the effect of low urate on neurological inflammation.

\section{vi. Acknowledgements}

This work was supported by Hatch Grant (WVA00678). Chickens were donated by Dr. Taylor (Morgantown, WV). Special thanks to Dr. Bowdridge, Dr. Matthew, and Dr. Yao for allowing me to use their facilities to conduct research. Specials thanks to Dr. Bowdridge and Dr. Garza for training and educating me in these numerous scientific methods to conduct my research. Special thanks to the West Virginia University farm crew for their help. Special thanks to Dr. Bidwai, Javiar Garza, Natalie Green, Brooke Zedar, Melissa Trani, Mohammad Al-Halak, Abdul Al-Halak, Joy Wang, Zach Phipps, Tom Kashiwagi and Liridon Marku for aiding in the 
research process from visiting the farm, writing up ideas, running experiments, review my work and giving me all the support to complete this thesis. In addition, I want to give Dr. Holaskova for encouraging me to work on the statistics, redo experiments to optimize the results and push me through the finish line. Lastly, thank you Hillar Klandorf for giving the opportunity to grow as a person, helping me build a new foundation, learn how to run a lab, meet all these great people and preform experiments that will aid the scientific community.

\section{vii. Literature Cited}

1. Bowman, Gene L, et al. "Uric Acid as a CNS Antioxidant." Journal of Alzheimer's Disease : JAD, U.S. National Library of Medicine, 2010, www.ncbi.nlm.nih.gov/pmc/articles/PMC2859185/. [8]

2. Chen, Juxing, et al. "Identification of Potential Biomarkers for Gut Barrier Failure in Broiler Chickens." Frontiers in Veterinary Science, Frontiers Media S.A., 26 May 2015, www.ncbi.nlm.nih.gov/pmc/articles/PMC4672187/. [2]

3. Elion, Gertrude B. "Allopurinol and Other Inhibitors of Urate Synthesis." SpringerLink, Springer, Berlin, Heidelberg, 1 Jan. 1978, link.springer.com/chapter/10.1007\%2F978-3642-66867-8_21. [5]

4. Fang, Pu, et al. "A Double-Edged Sword: Uric Acid and Neurological Disorders." Brain Disorders \& Therapy, U.S. National Library of Medicine, 1 Nov. 2013, www.ncbi.nlm.nih.gov/pmc/articles/PMC3914730/. [12]

5. George, Jacob, et al. "High-Dose Allopurinol Improves Endothelial Function by Profoundly Reducing Vascular Oxidative Stress and Not by Lowering Uric Acid.” Circulation, vol. 114, no. 23, 2006, pp. 2508-2516., doi:10.1161/circulationaha.106.651117. [5]

6. Gersch, et al. "Uric Acid and the Immune Response *." OUP Academic, Oxford 
University Press, 24 July 2006, academic.oup.com/ndt/article/21/11/3046/1872009. [2]

7. Ghaemi-Oskouie, Faranak, and Yan Shi. "The Role of Uric Acid as an Endogenous Danger Signal in Immunity and Inflammation." Current Rheumatology Reports, U.S. National Library of Medicine, Apr. 2011, www.ncbi.nlm.nih.gov/pmc/articles/PMC3093438/. [1]

8. Heo, Sung Hyuk, and Seung-Hoon Lee. "High Levels of Serum Uric Acid Are Associated with Silent Brain Infarction.” Journal of the Neurological Sciences, Elsevier, 3 Aug. 2010, www.sciencedirect.com/science/article/pii/S0022510X10003084. [8]

9. Hong, Yeong Ho, et al. "Analysis of Chicken Cytokine and Chemokine Gene Expression Following Eimeria Acervulina and Eimeria Tenella Infections.” Veterinary Immunology and Immunopathology, Elsevier, 20 Sept. 2006, WwW.sciencedirect.com/science/article/pii/S0165242706002017. [14]

10. Hong, Yeong Ho, et al. "Changes in Immune-Related Gene Expression and Intestinal Lymphocyte Subpopulations Following Eimeria Maxima Infection of Chickens.” Veterinary Immunology and Immunopathology, Elsevier, 12 Oct. 2006, WWW.sciencedirect.com/science/article/pii/S0165242706002376. [15]

11. Houser, Madelyn C., and Malú G. Tansey. "The Gut-Brain Axis: Is Intestinal Inflammation a Silent Driver of Parkinson's Disease Pathogenesis?” Nature News, Nature Publishing Group, 11 Jan. 2017, www.nature.com/articles/s41531-016-0002-0. [7]

12. Keith W. Jarosinski Bradley L. Njaa Priscilla H. O'connellDr, et al. "ProInflammatory Responses in Chicken Spleen and Brain Tissues after Infection with Very Virulent Plus Marek's Disease Virus.” Mary Ann Liebert, Inc., Publishers, 31 Mar. 2005, www.liebertpub.com/doi/abs/10.1089/vim.2005.18.148. [16] 
13. Martinon, Fabio. "Update on Biology: Uric Acid and the Activation of Immune and Inflammatory Cells.” SpringerLink, Current Science Inc., 11 Mar. 2010, link.springer.com/article/10.1007\%2Fs11926-010-0092-3. [4]

14. Neuman, Manuela G, et al. "Biomarkers in Nonalcoholic Fatty Liver Disease." Canadian Journal of Gastroenterology \& Hepatology, Pulsus Group Inc, Dec. 2014, www.ncbi.nlm.nih.gov/pmc/articles/PMC4277175/. [17]

15. "Oxidative Stress with an Activation of the... : Journal of Hypertension." $L W W$, journals.lww.com/jhypertension/Abstract/2010/06000/Oxidative_stress_with_an_activati on_of_the.18.aspx. [6]

16. "Pathology of Pet and Aviary Birds." Wiley Online Library, onlinelibrary.wiley.com/book/10.1002/9780470376836. [9]

17. Sautin, Yuri Y, and Richard J Johnson. "Uric Acid: the Oxidant-Antioxidant Paradox." Nucleosides, Nucleotides \& Nucleic Acids, U.S. National Library of Medicine, June 2008, www.ncbi.nlm.nih.gov/pmc/articles/PMC2895915/. [10]

18. Shi, Yan. "Caught Red-Handed: Uric Acid Is an Agent of Inflammation.” The Journal of Clinical Investigation, American Society for Clinical Investigation, June 2010, www.ncbi.nlm.nih.gov/pmc/articles/PMC2877961/. [3]

19. Sun, Xiao, et al. "Arsenic Affects Inflammatory Cytokine Expression in Gallus Brain Tissues.” SpringerLink, BioMed Central, 5 June 2017, link.springer.com/article/10.1186/s12917-017-1066-8. [18]

20. Viña, José, et al. "Mechanism of Free Radical Production in Exhaustive Exercise in Humans and Rats; Role of Xanthine Oxidase and Protection by Allopurinol." IUBMB, John Wiley \& Sons, Ltd, 18 Jan. 2008, 
iubmb.onlinelibrary.wiley.com/doi/pdf/10.1080/15216540050167098. [11]

21. Winklewski, Pawel J, et al. "Brain Inflammation and Hypertension: the Chicken or the Egg?" Journal of Neuroinflammation, BioMed Central, 3 May 2015, jneuroinflammation.biomedcentral.com/articles/10.1186/s12974-015-0306-8. [13]

22. Bar Shira, Enav, and Aharon Friedman. "Innate Immune Functions of Avian Intestinal Epithelial Cells: Response to Bacterial Stimuli and Localization of Responding Cells in the Developing Avian Digestive Tract." PloS One, Public Library of Science, 6 July 2018, www.ncbi.nlm.nih.gov/pmc/articles/PMC6034880/. [21]

23. Minghetti, Luisa. "Cyclooxygenase-2 (COX-2) in Inflammatory and Degenerative Brain Diseases.” Journal of Neuropathology and Experimental Neurology, U.S. National Library of Medicine, Sept. 2004, www.ncbi.nlm.nih.gov/pubmed/15453089. [27]

24. Rothaug, Michelle, et al. "The Role of Interleukin-6 Signaling in Nervous Tissue." Biochimica Et Biophysica Acta (BBA) - Molecular Cell Research, Elsevier, 23 Mar. 2016, www.sciencedirect.com/science/article/pii/S0167488916300714. [25]

25. Wigley, and Kaiser. "Avian Cytokines in Health and Disease.” Brazilian Journal of Poultry Science, Fundação APINCO De Ciência e Tecnologia Avícolas, www.scielo.br/scielo.php?script=sci_arttext\&amp;pid=S1516-635X2003000100001. [22]

26. Lewis, Jeffrey R., and Smruti R. Mohanty. "Nonalcoholic fatty liver disease: a review and update." Digestive diseases and sciences 55.3 (2010): 560-578. [19]

27. Sarin, Shiv K., and Ashok Choudhury. "Acute-on-chronic liver failure: terminology, mechanisms and management." Nature Reviews Gastroenterology \& Hepatology 13.3 (2016): 131. [20]

28. Grant, Lafaine M., and Don C. Rockey. "Drug-induced liver injury." Current opinion in 
gastroenterology 28.3 (2012): 198-202. [24]

29. Patrignani, Paola, et al. "New insights into COX-2 biology and inhibition." Brain Research Reviews 48.2 (2005): 352-359. [28]

30. Giovannini, M. G., et al. "Experimental brain inflammation and neurodegeneration as model of Alzheimer's disease: protective effects of selective COX-2 inhibitors." International journal of immunopathology and pharmacology 16.2; (2003): 31-40. [29]

31. Wang, Y., Du, W., Lei, K. et al. Effects of Dietary Bacillus licheniformis on Gut Physical Barrier, Immunity, and Reproductive Hormones of Laying Hens. Probiotics \& Antimicro. Prot. 9, 292-299 (2017). https://doi.org/10.1007/s12602-017-9252-3. [23] 
A

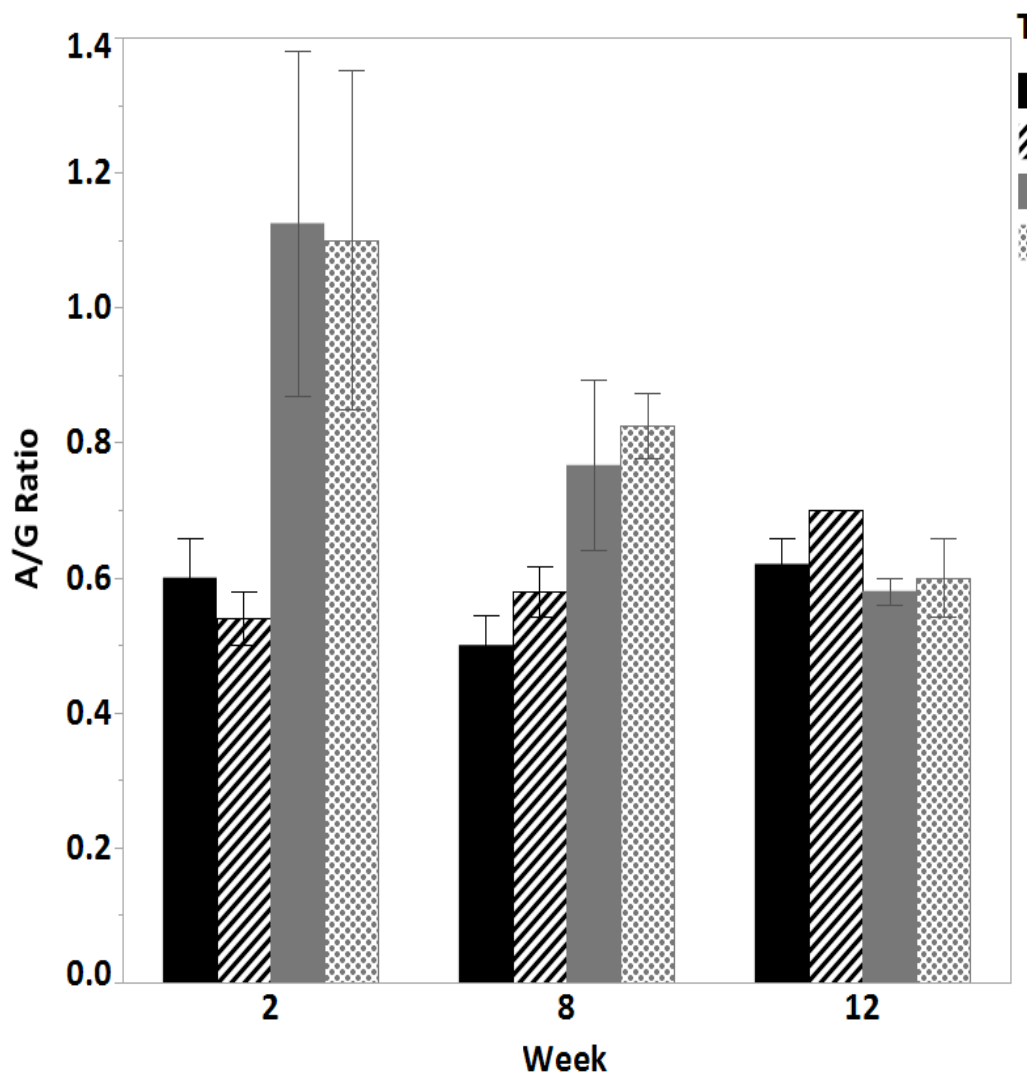

Treatment combination

Control_M V/Allopurinol_M

Control_F

Allopurinol_F
B

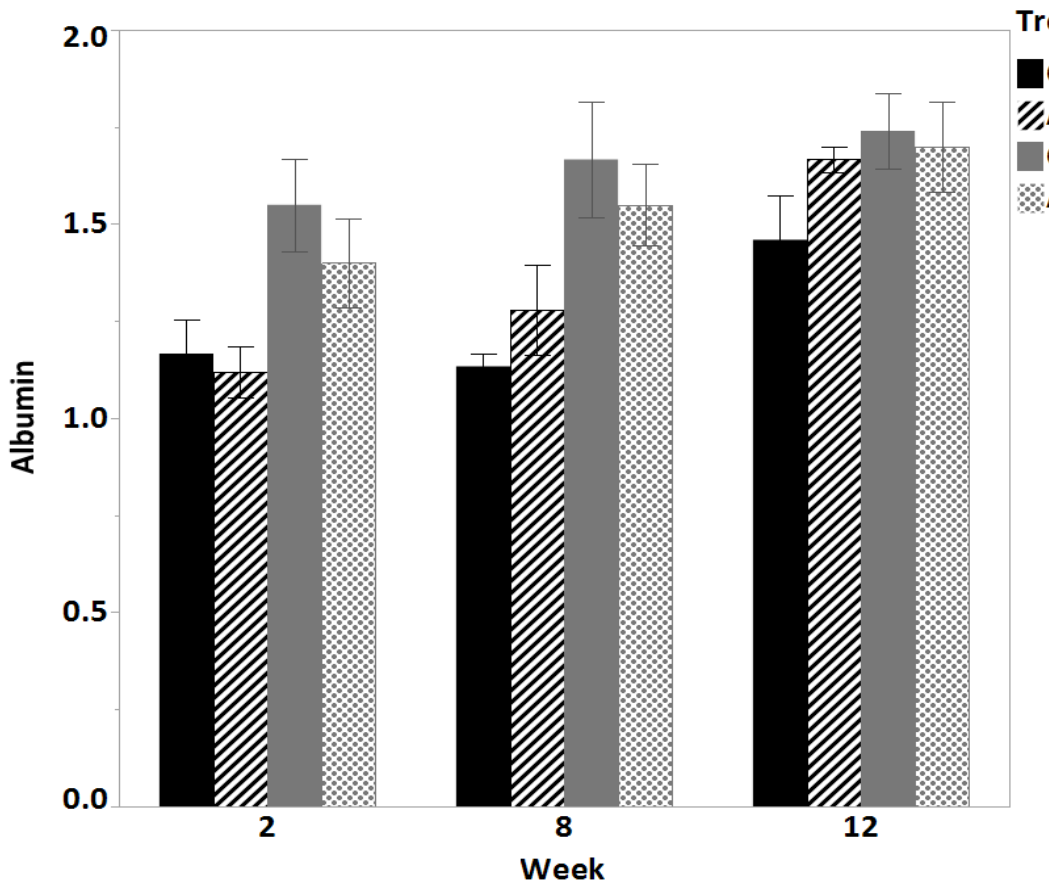

Treatment combination

Control_M

Y/Allopurinol_M

Control_F

Allopurinol_F 
C

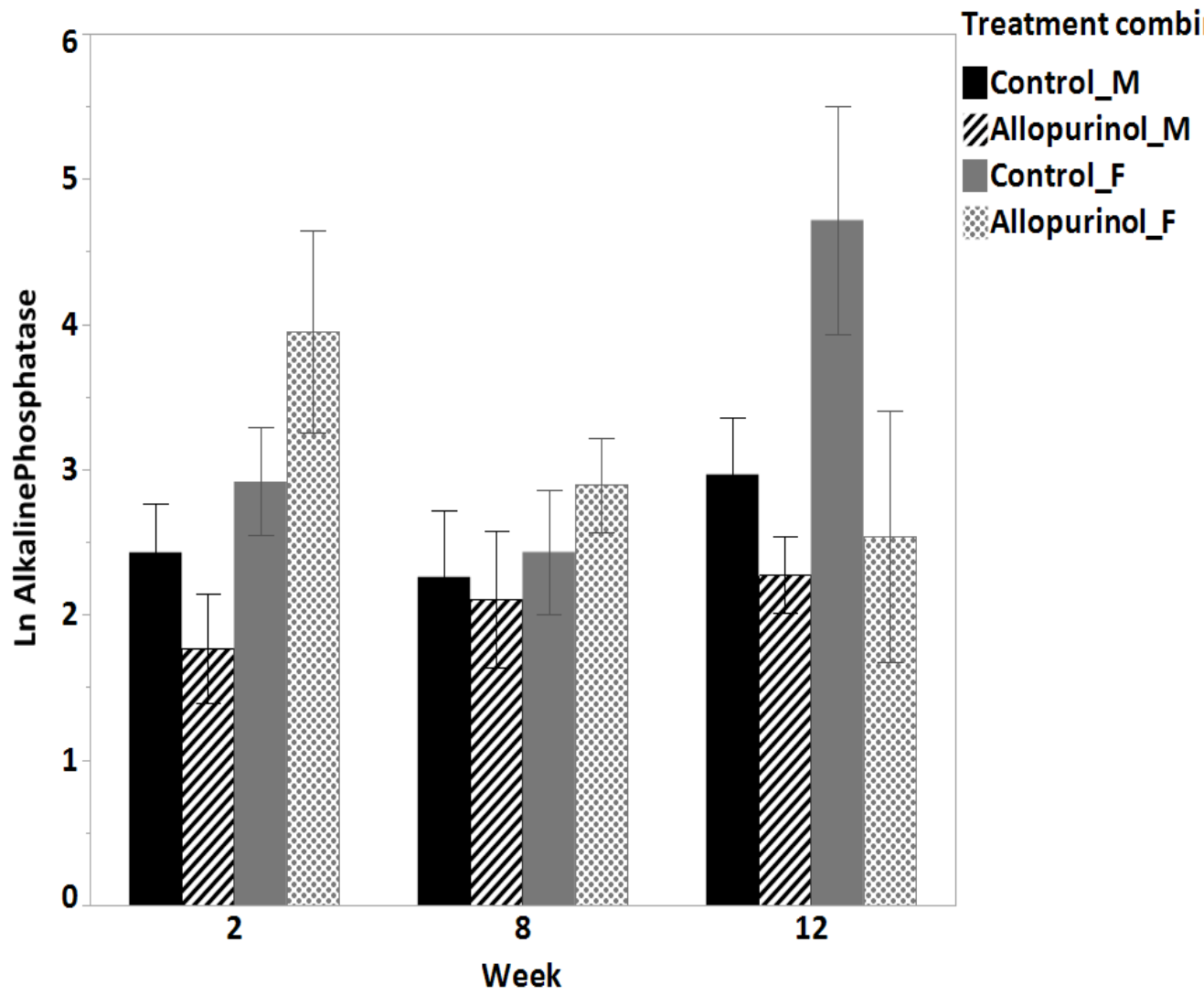

D

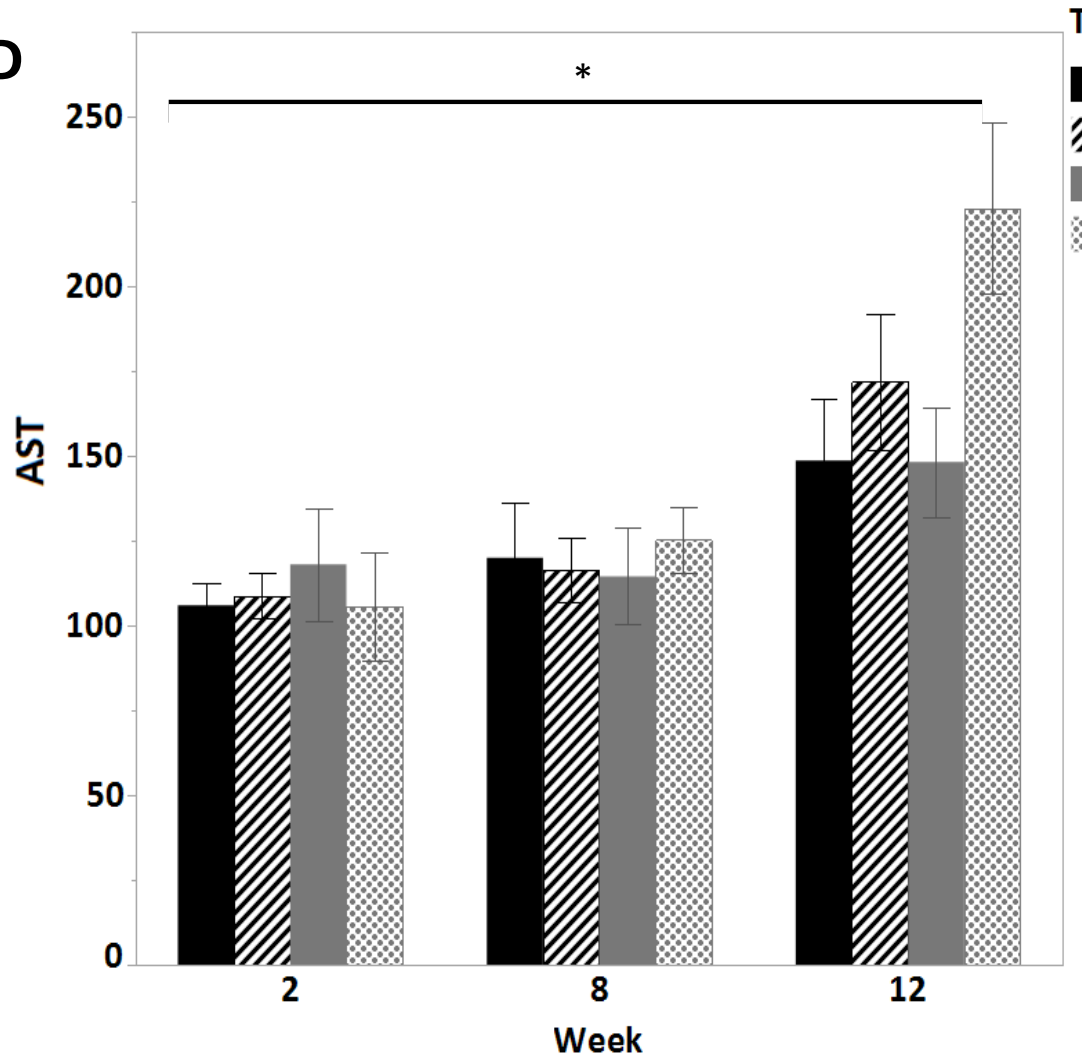

Treatment combination

Control_M

Y,Allopurinol_M

Control_F

Allopurinol_F 
E

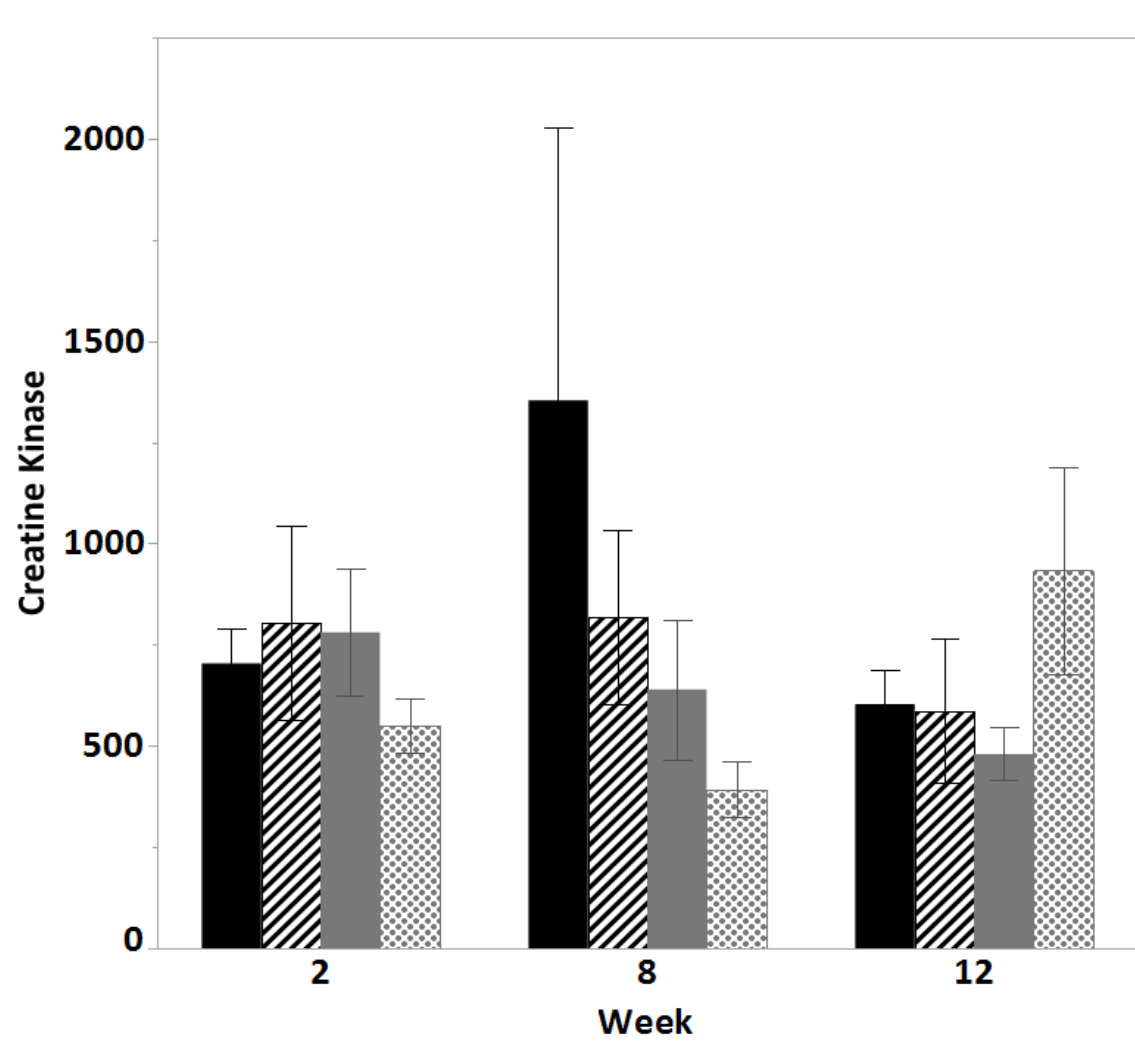

Treatment combination

口Control_M

YAAllopurinol_M

Control_F

Allopurinol_F

F

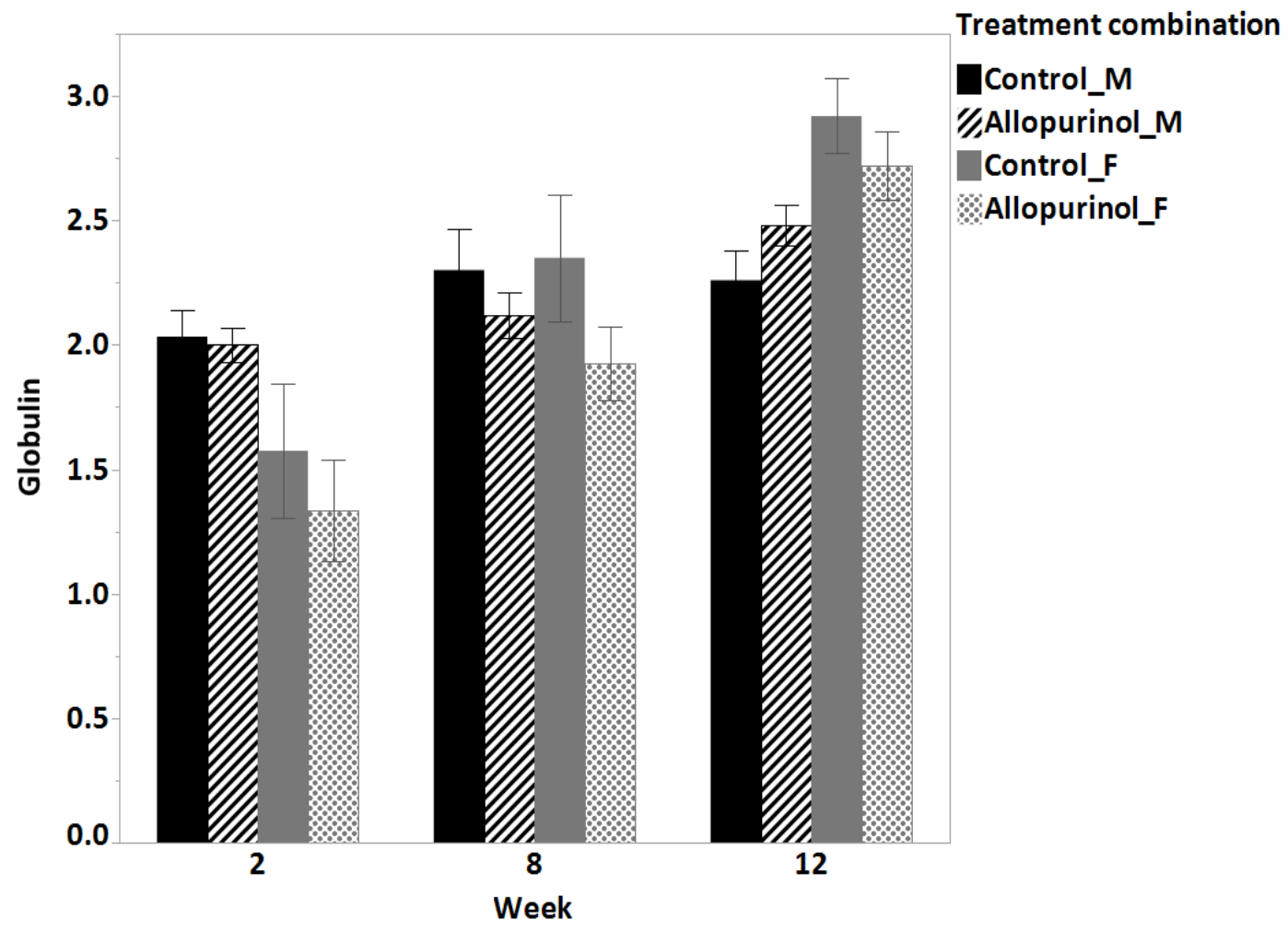


G

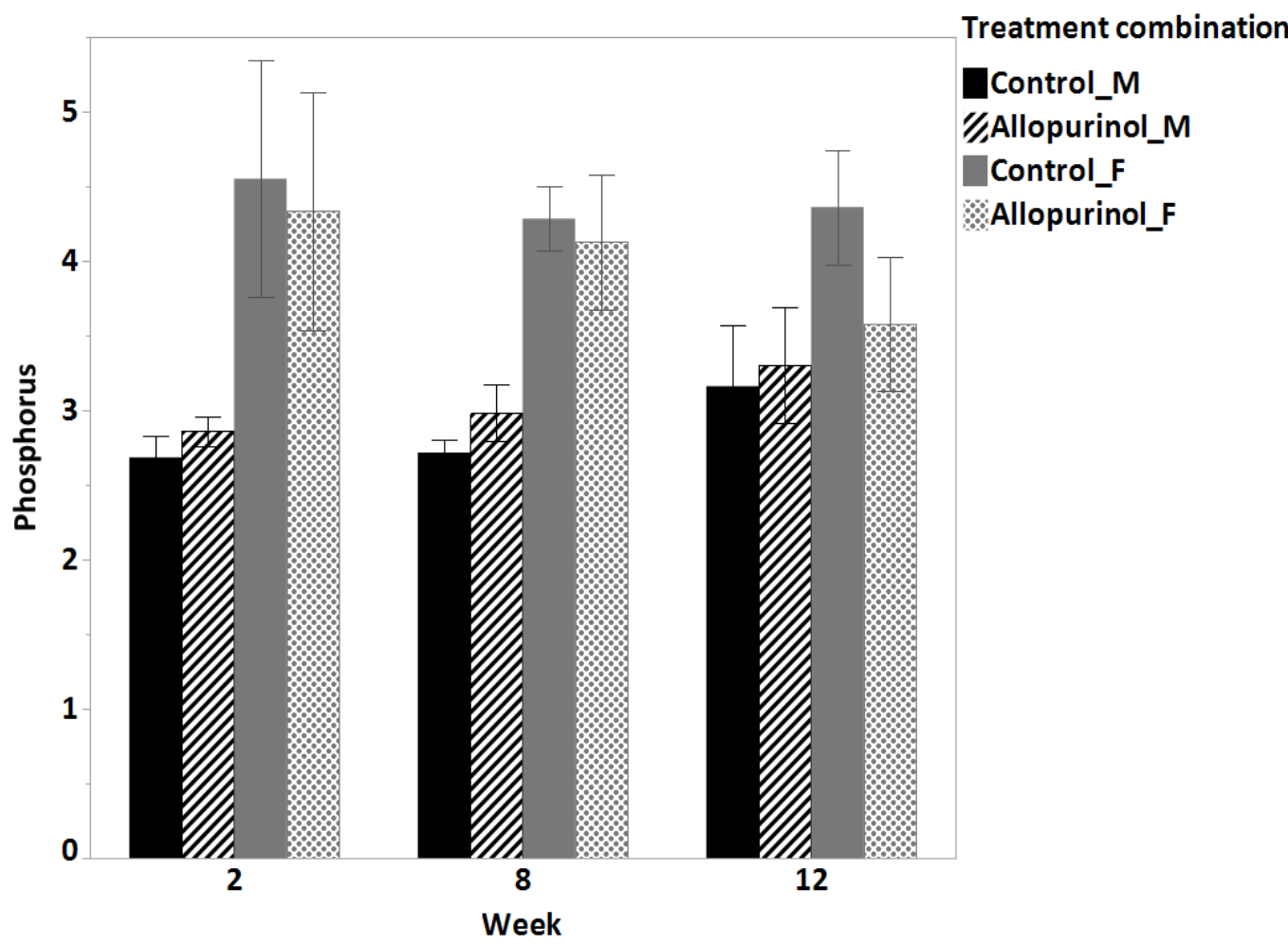

H

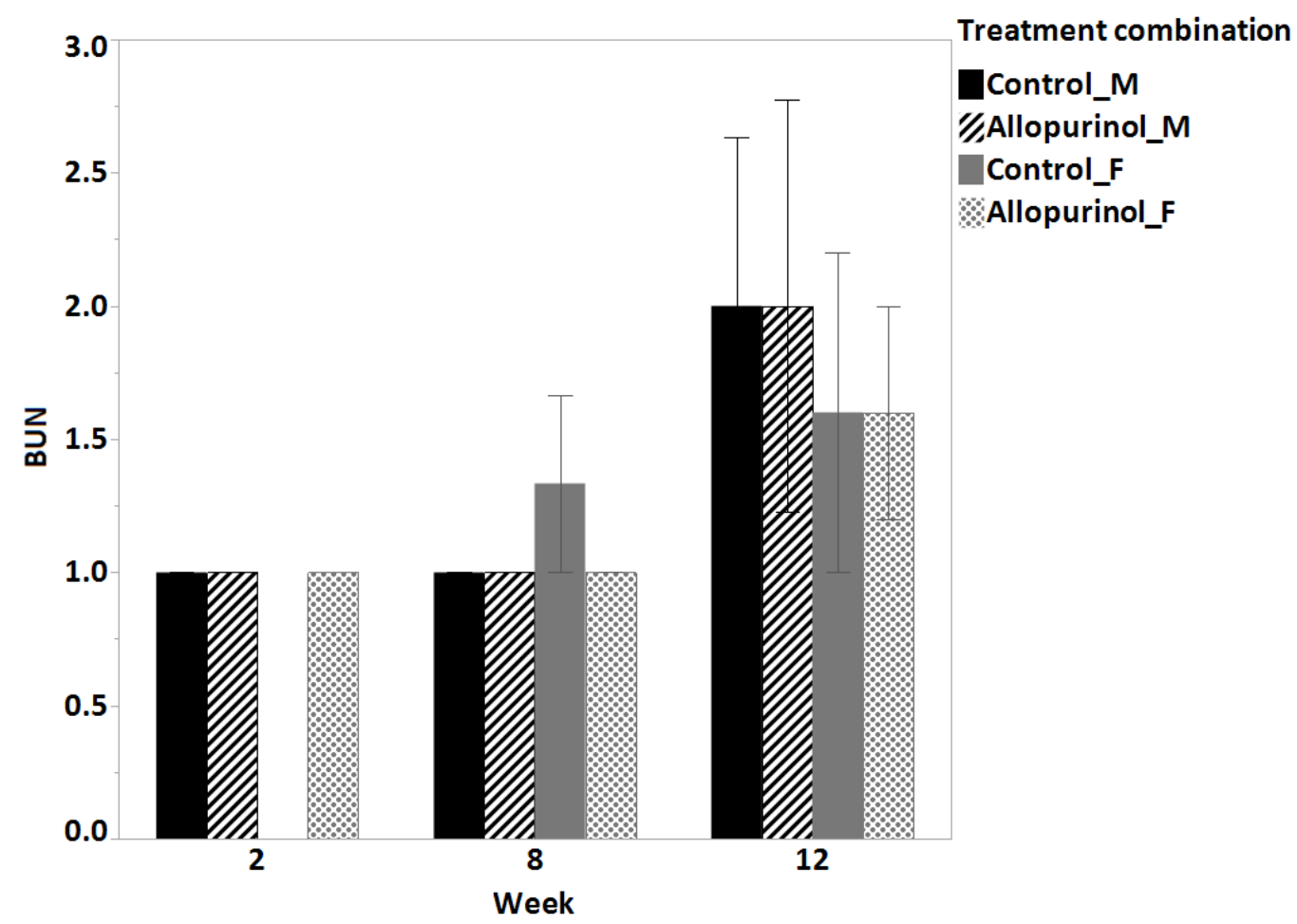




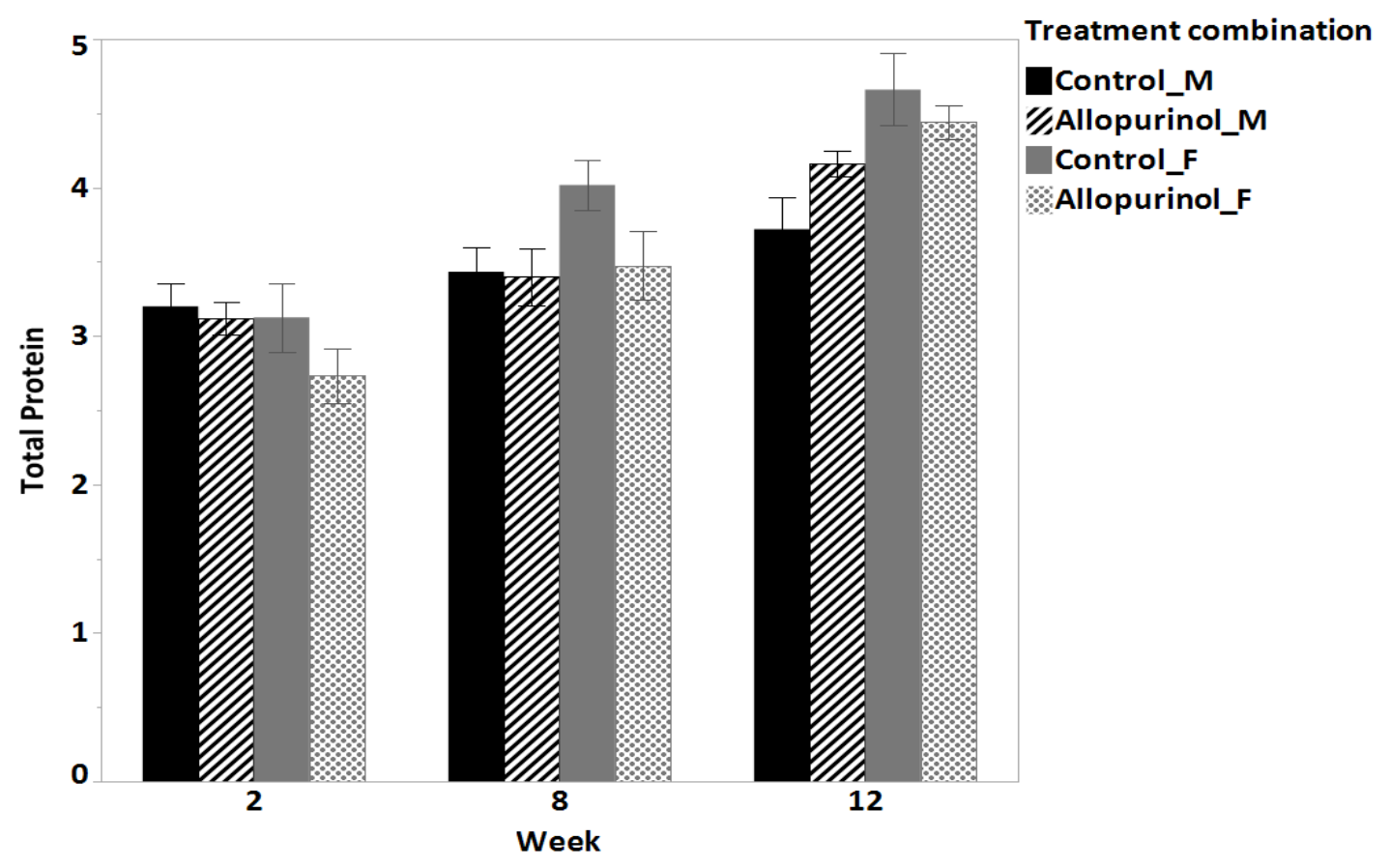

J

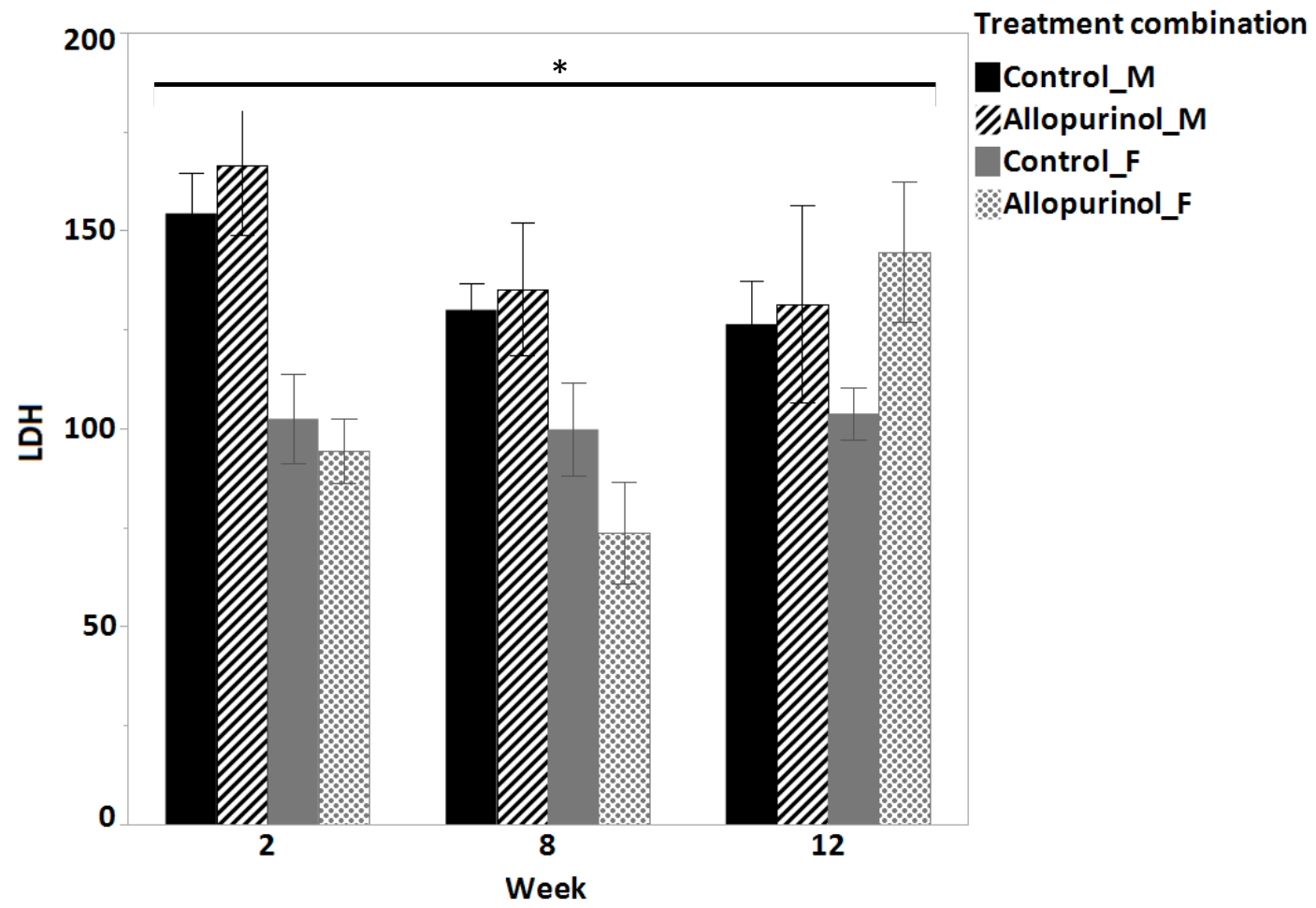


K

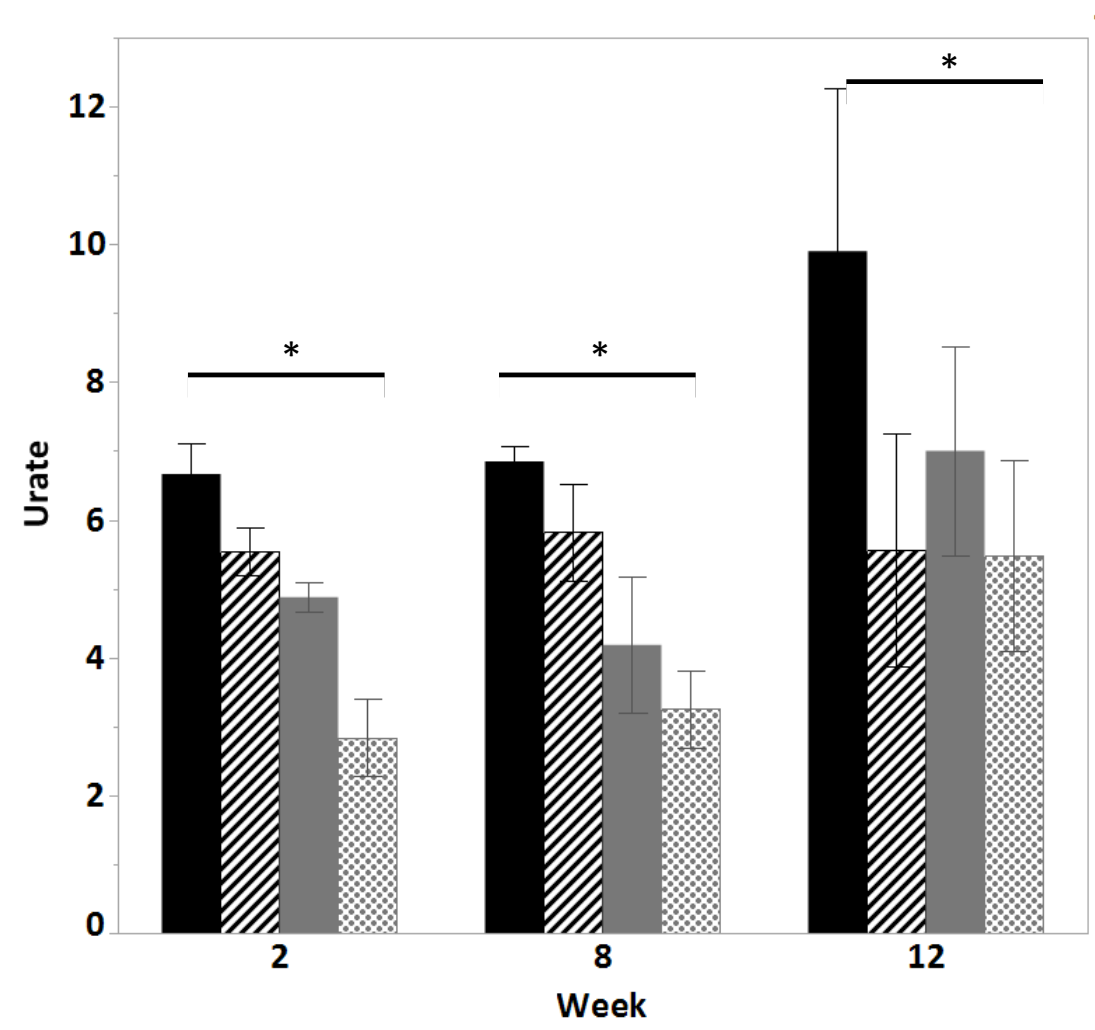

Treatment combination

Control_M

YAllopurinol_M

Control_F

Allopurinol_F

L

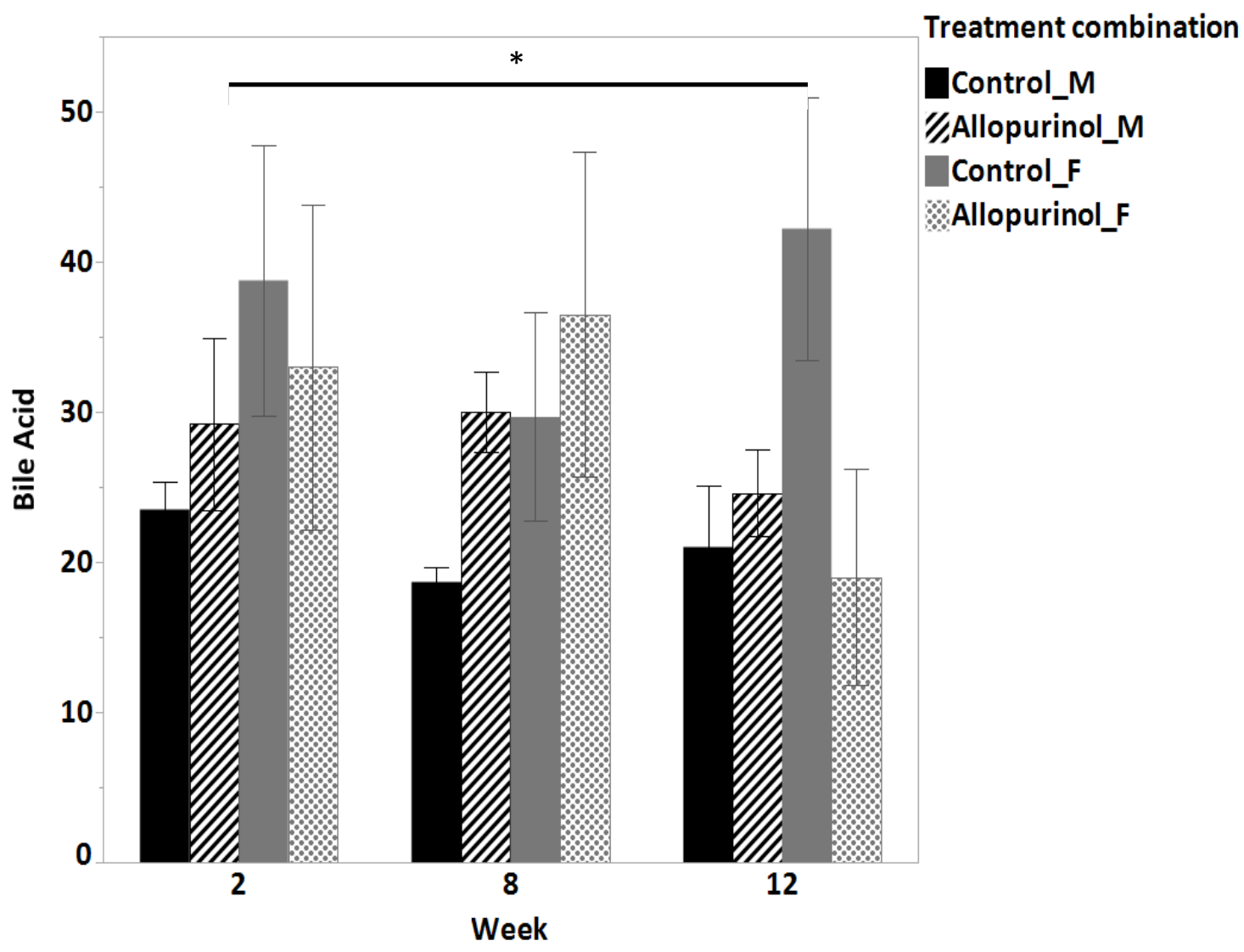




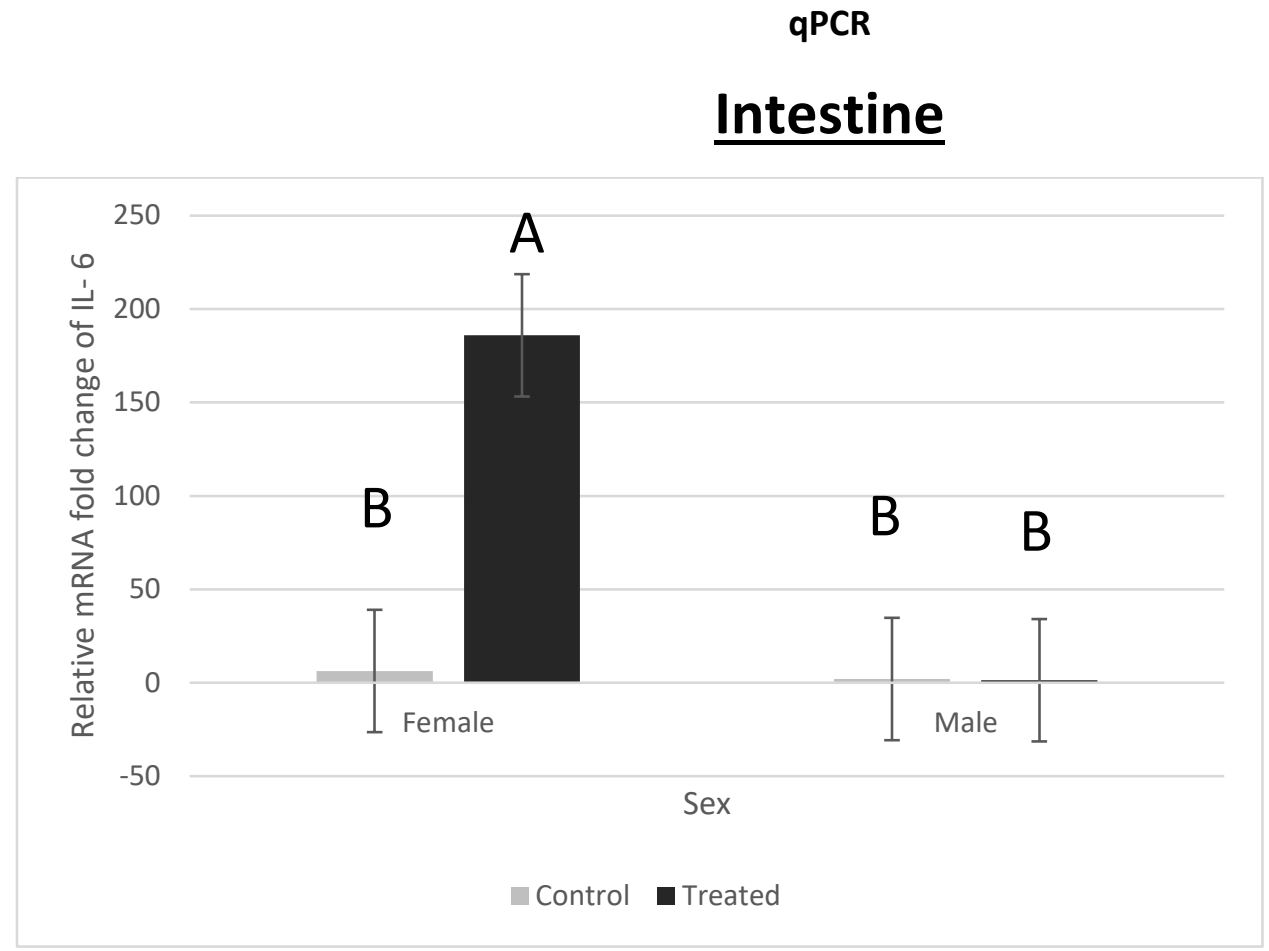

Figure 2a. Relative expression levels of IL-6 in the intestines.

The effect of allopurinol on female chickens for IL-6 expression is statistically increased from every other group by 185.93 times.

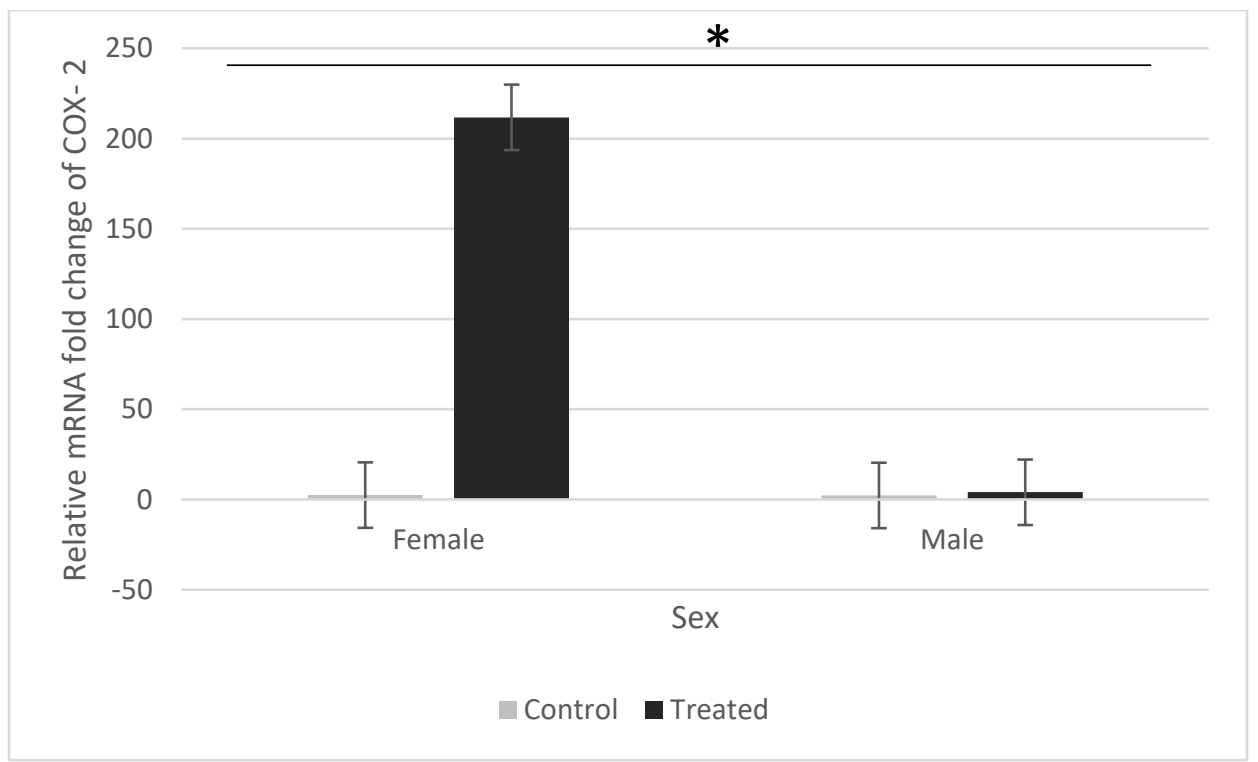

Figure 2b. Relative expression levels of COX-2 in the intestines. 
The effect of allopurinol on female chickens for COX-2 expression is statistically increased from every other group by 211.74 times.

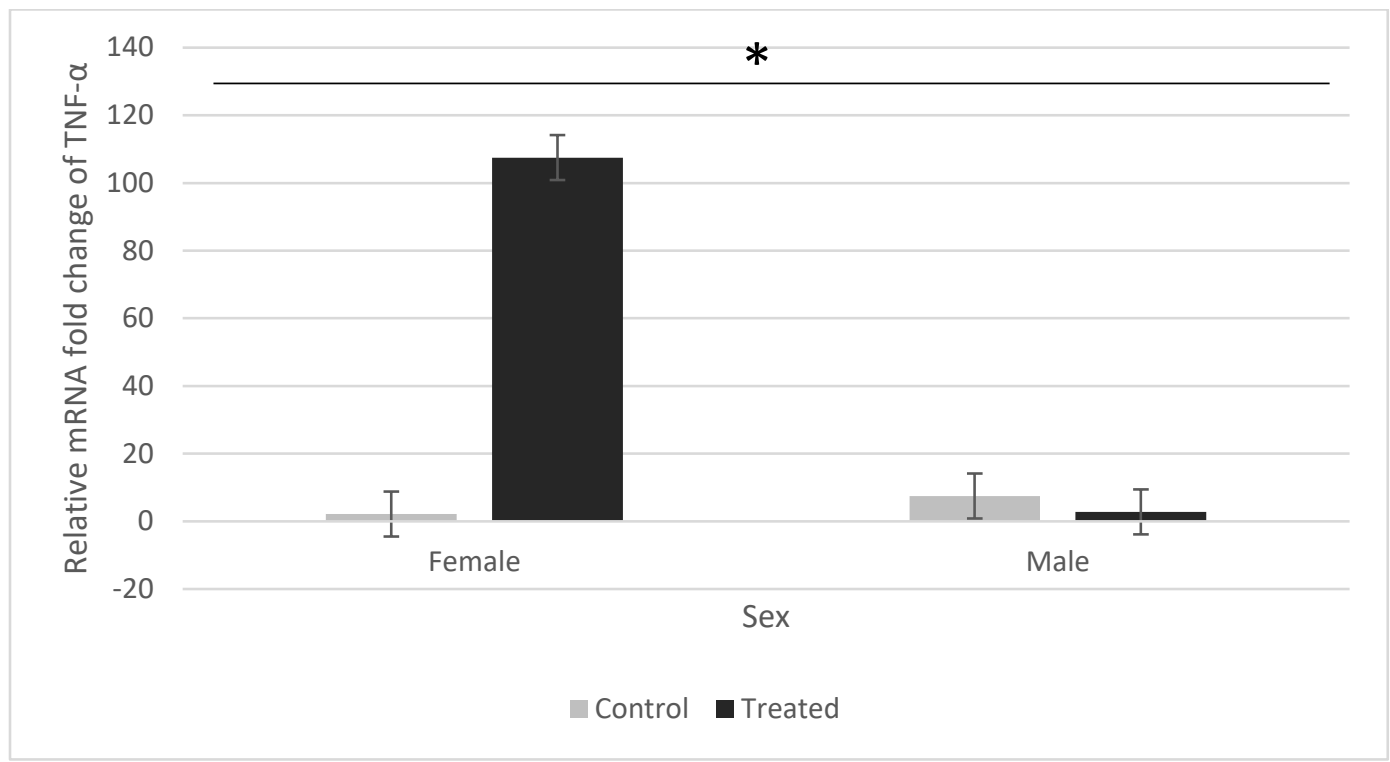

Figure 2c. Relative expression levels of TNF- $\alpha$ in the intestines.

The effect of allopurinol on female chickens for TNF- $\alpha$ expression is statistically increased from every other group by 107.52 times. 


\section{Liver}

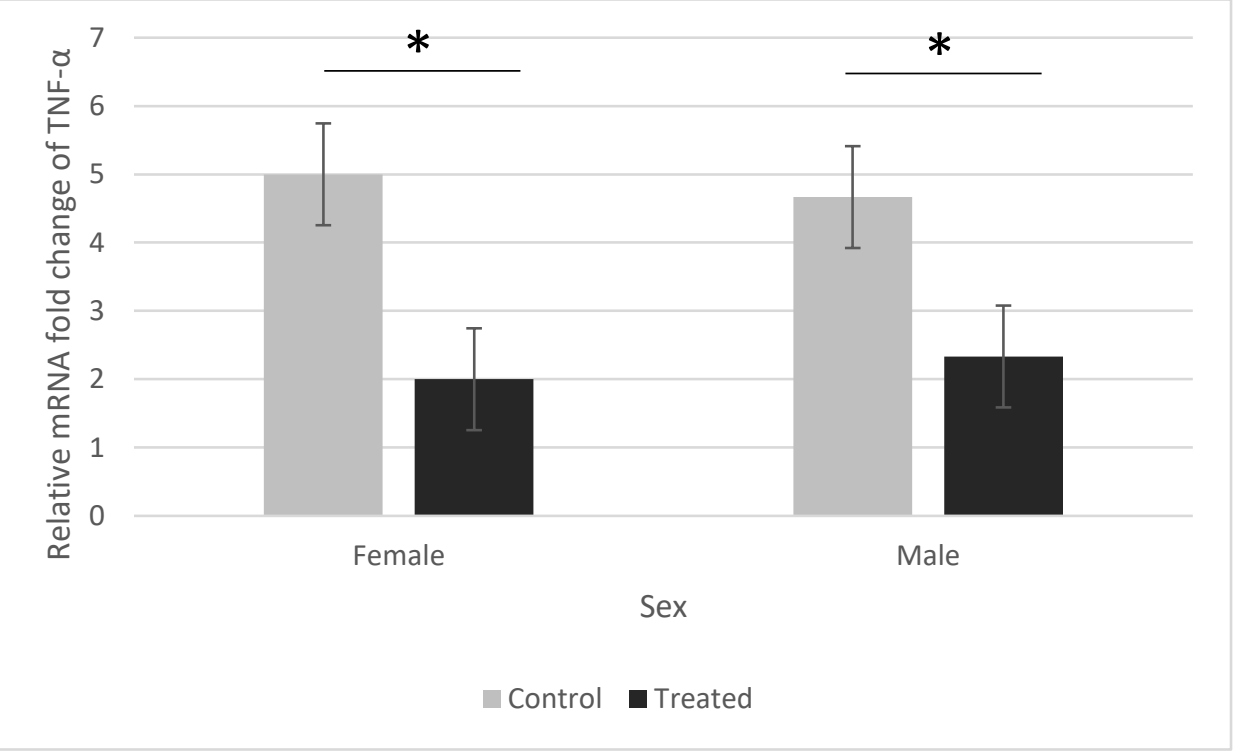

Figure 3a. Relative expression levels of TNF- $\alpha$ in the liver.

The effect of allopurinol on TNF- $\alpha$ expression has statistically decreased in both female and male by 3 and 2 times from the control groups respectively.

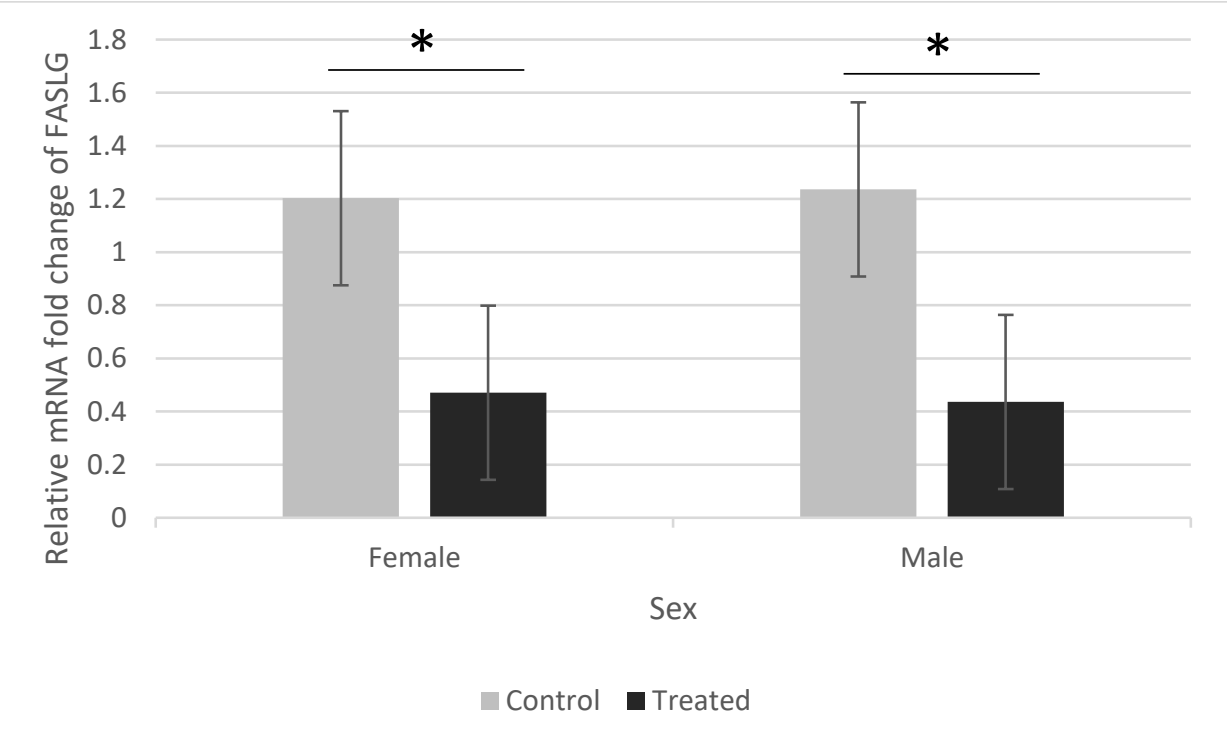

Figure 3b. Relative expression levels of FASLG in the liver. 
The effect of allopurinol on FASLG expression has statistically decreased by 2 times in both male and females from the control group respectively.

\section{Midbrain}

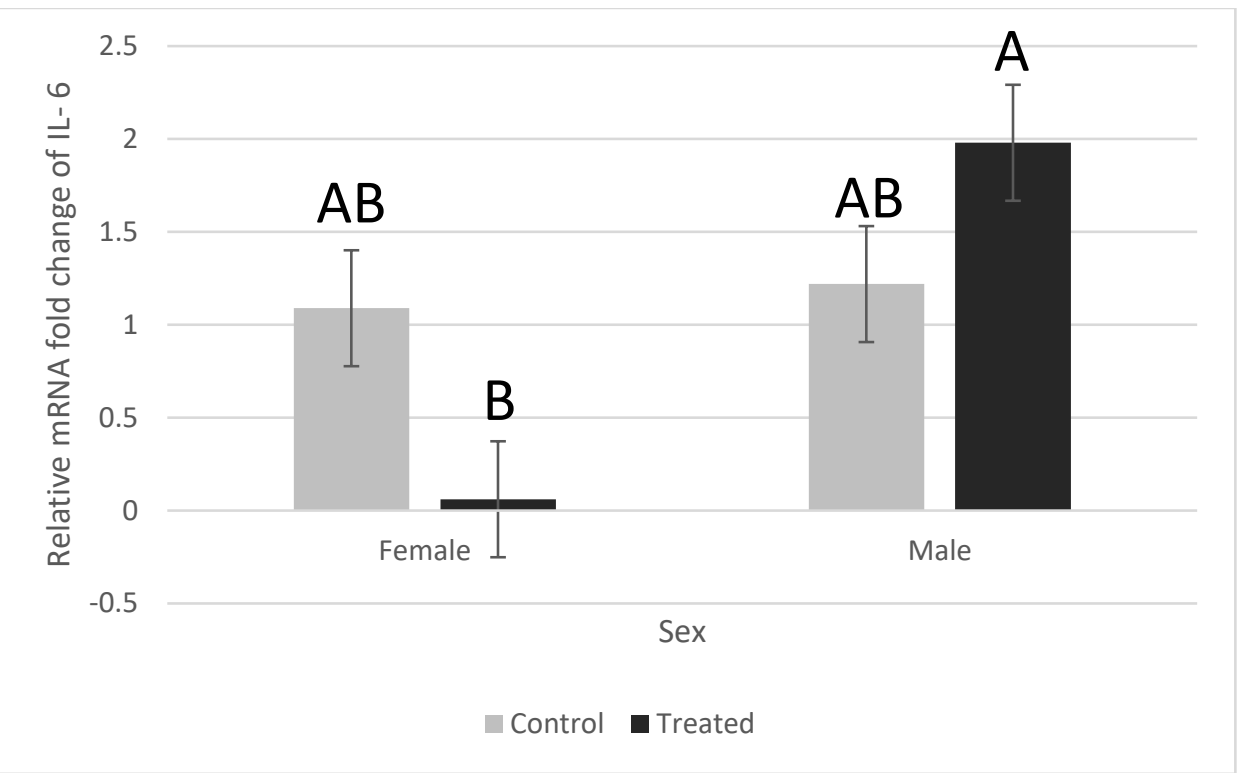

Figure 4a. Relative expression levels of IL-6 in the midbrain. The effect of allopurinol on female chickens on IL-6 expression has statistically decreased from its respective control with an $\mathrm{LSM}=0.061$ and $\mathrm{SE}=0.0312$. The effect of allopurinol on male chickens on IL-6 expression has statistically increased from its respective control group $\mathrm{LSM}=1.98$ and $\mathrm{SE}=\mathbf{0 . 0 3 1 2}$. 


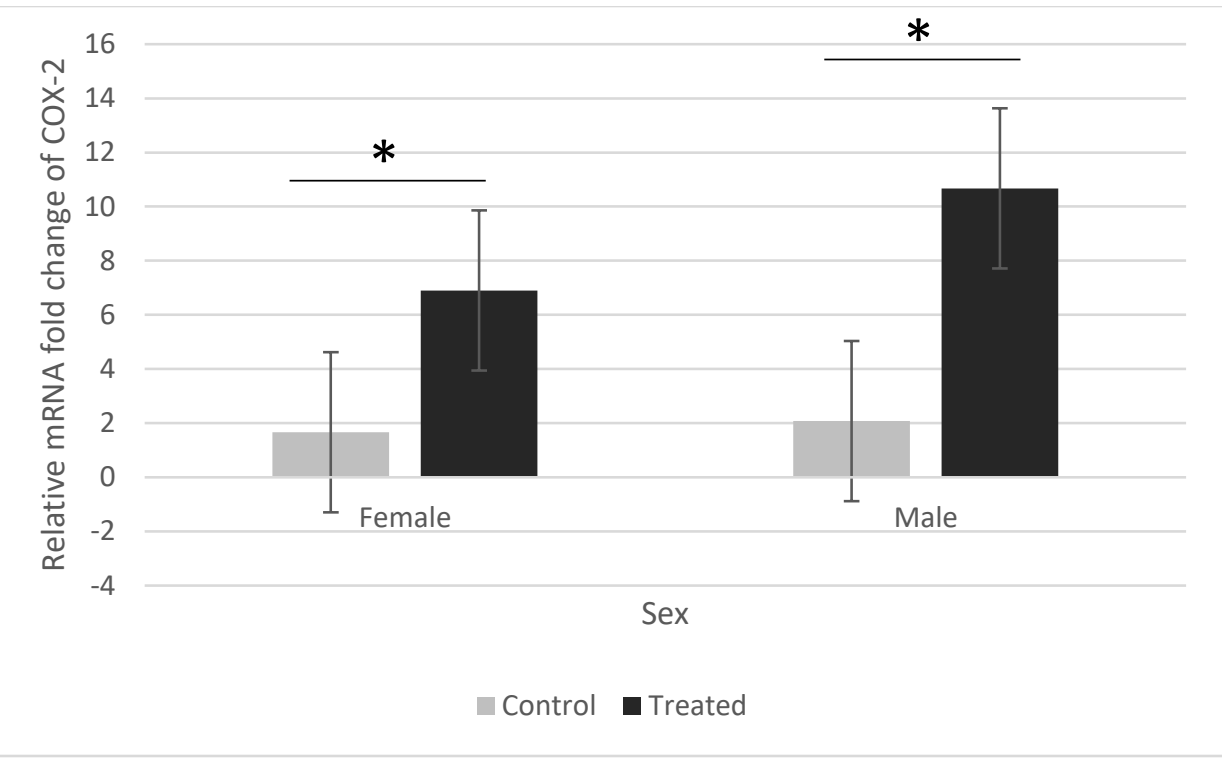

Figure 4b. Relative expression levels of COX-2 in the midbrain.

The effect of allopurinol on COX-2 expression has statistically increased in females and males by 7 and 10 times from the control group respectively. 